\title{
Star-spot distributions and chromospheric activity on the RS CVn type eclipsing binary SV Cam
}

\author{
H. V. Şenavc1, ${ }^{1 \star}$ E. Bahar, ${ }^{1 \star}$ D. Montes, ${ }^{2}$ S. Zola, ${ }^{3}$ G. A. J. Hussain, ${ }^{4}$ A. Frasca, ${ }^{5}$ \\ E. Iş1 $\mathrm{k}^{6,7 \star}$ and $\mathrm{O}$. Yörükoğlu ${ }^{1}$ \\ ${ }^{1}$ Department of Astronomy and Space Sciences, Faculty of Science, Ankara University, Tandogan, 06100 Ankara, Turkey \\ ${ }^{2}$ Dpto. Astrofisica, Facultad de CC. Fisicas, Universidad Complutense de Madrid, E-28040 Madrid, Spain \\ ${ }^{3}$ Astronomical Observatory, Jagiellonian University, ul. Orla 171, PL-30-244 Krakow, Poland \\ ${ }^{4}$ European Southern Observatory, Karl-Schwarzschild-Str 2, D-85748 Garching bei München, Germany \\ ${ }^{5}$ INAF - Osservatorio Astrofisico di Catania, via S. Sofia, 78, I-95123 Catania, Italy \\ ${ }^{6}$ Max-Planck-Institut für Sonnensystemforschung, Justus-von-Liebig-Weg 3, D-37077 Göttingen, Germany \\ ${ }^{7}$ Feza Gürsey Center for Physics and Mathematics, Boğaziçi University, Kuleli/Üsküdar, 34684 Istanbul, Turkey
}

Accepted 2018 June 1. Received 2018 June 1; in original form 2018 February 6

\begin{abstract}
Using a time series of high-resolution spectra and high-quality multicolour photometry, we reconstruct surface maps of the primary component of the RS CVn type rapidly rotating eclipsing binary, SV Cam (F9V + K4V). We measure a mass ratio, q, of 0.641(2) using our highest quality spectra and obtain surface brightness maps of the primary component, which exhibit predominantly high-latitude spots located between $60^{\circ}$ and $70^{\circ}$ latitudes with a mean filling factor of $\sim 35$ per cent. This is also indicated by the $R$-band light-curve inversion, subjected to rigorous numerical tests. The spectral subtraction of the $\mathrm{H} \alpha$ line reveals strong activity of the secondary component. The excess $\mathrm{H} \alpha$ absorption detected near the secondary minimum hints to the presence of cool material partially obscuring the primary star. The flux ratios of CaII IRT excess emission indicate that the contribution of chromospheric plage regions associated with star-spots is dominant, even during the passage of the filament-like absorption feature.
\end{abstract}

Key words: stars: activity - binaries: eclipsing-stars: imaging.

\section{INTRODUCTION}

RS CVn-type binaries are composed of F-K type dwarf/giant components. Their orbital periods most typically range from 1 to $20 \mathrm{~d}$. Systems with smaller orbital periods exhibit strong magnetic activity, which is thought to be related to rapid rotation. They offer laboratories to study stellar activity in post-main-sequence stars influenced by tidal effects (Strassmeier 2009). The presence of cool spots on eclipsing RS CVn-type systems is responsible for significant variability in their light curves outside eclipses (Berdyugina 2005). Their effects can also be detected in spectral line cores that form in the chromosphere, e.g. CaII $\mathrm{H} \& \mathrm{~K}$ and $\mathrm{H} \alpha$, where core emission can be much stronger than in the Sun (Strassmeier et al. 2000).

SV Cam is a detached close binary of RS CVn-type (HD 44982, $m_{v}=8.40$ ), with an orbital period of $\sim 0.593 \mathrm{~d}$. There have been several estimates of its spectral type, ranging from F9V to G2V for the primary component and $\mathrm{K} 2 \mathrm{~V}$ to $\mathrm{K} 7 \mathrm{~V}$ for the secondary,

^E-mail: hvsenavci@ankara.edu.tr (HVS); enbahar@ankara.edu.tr (EB); respectively, the most recent one being F9V + K4V by Jeffers et al. (2006b). The latest investigation on the orbital period variation of SV Cam was also performed by Manzoori (2016), indicating two cyclic variations that are interpreted as light-time effect due to the existence of a third body and magnetic activity cycle of the system. There are also several studies in the literature related to the activity behaviour of SV Cam. Based on photometric data spanning over 50 years, Zeilik et al. (1988) pointed out that the spot groups on the primary component fall into two zones as active longitude belts. Using the photometric observations made by Patkos (1982) during the 1973-1981 period, Djurasevic (1998) performed spot modelling and mentioned the existence of active longitudes. In a series of papers, Zboril \& Djurašević (2003); Zboril \& Djuraševič (2004, 2006) monitored the brightness variation of the system outside eclipses using data obtained between 2001 and 2005, and also argued about the existence of two spots on the primary component, but with varying dimensions and locations from one season to another. Hempelmann et al. (1997) obtained Doppler images of the system using Ca I (6103, 6122, and $6439 \AA)$ and Fe I (6400 ̊) lines. The surface maps obtained in that study revealed a dominant spot region located at around $40-75^{\circ}$ longitudes and latitudes between 
+30 and $60^{\circ}$. Rucinski et al. (2002) determined the mass ratio of the system as $q=M_{2} / M_{1}=0.641$ and the spectral type of the primary component as G2V. They also determined the masses of the primary and the secondary components as 1.14 and $0.73 \mathrm{M}_{\odot}$, respectively. With the help of spectroscopic observations covering the entire orbital period of SV Cam, Kjurkchieva, Marchev \& Zola (2002) determined the mass ratio of the system as $q=M_{2} / M_{1}=$ 0.593 . Their results indicated the presence of two cool spots on the primary component, with their maximum visibilities at phases 0.27 and 0.86 .

A series of papers concerning the star-spot distribution in $\mathrm{SV}$ Cam were published by Jeffers et al. (2005, 2006a,b). In Jeffers et al. (2005), they modelled the spectrophotometric Hubble Space Telescope (HST) data of SV Cam using PHOENIX model atmospheres and found that the surface flux in the eclipsed low-latitude region of the primary is about 30 per cent lower than that computed from the models. They also investigated the full surface flux of the primary component concluding that there is an additional flux deficit from the entire primary, which can be explained via a large polar spot on this star, extending from the pole to the latitude of $\sim 48^{\circ}$. Based on the spectrophotometric HST observations, they determined the effective temperatures of the primary and secondary components to be $T_{1}=6013 \mathrm{~K}$ and $T_{2}=4804 \mathrm{~K}$, respectively. The study by Jeffers et al. (2006a) includes an eclipse-mapping of the primary component, aimed at determination of the filling factors and sizes distribution of star-spots, too small to be resolved by DI. In addition to the HST data, they used ground-based photometric observations, in order to obtain the light curve of the system covering the full orbital cycle. Taking the advantage of high precision HST data, the detected strong discontinuities at the four contact points in the residuals of the fit to the light curve can only be removed by the reduction of the photospheric temperature and presence of a polar spot. They concluded that the spottedness of the stellar surface can have a significant impact on the determination of the stellar parameters, such as radius and effective temperature, in the components of a binary like SV Cam case. Using the first and second derivatives of the HST data, Jeffers et al. (2006b) determined the best-fitting atmosphere model by adjusting the models to the brightness variations during the primary eclipse. They also emphasized the importance of other stellar parameters such as limb darkening coefficients, as they can alter the intensity values across the stellar disc.

The most recent activity-related study of the system was performed by Manzoori (2016), who analysed the AAVSO (American Association of Variable Star Observers) 2006-2009 light curves of SV Cam. He mentioned that the star-spots appear at high latitudes on both components of SV Cam. He obtained cyclic orbital period variations with periods of 23.3 and 20.2 years from the $O$ - $C$ analysis. Furthermore, the Fourier transform of the second quadrature brightness variations led to another possible periodicity of $\sim 35$ years.

In this work, we aim to study the activity behaviour of SV Cam, combining several widely used tools together for the first time. We analyse the time-series high-resolution spectroscopic data via Doppler imaging, spectral synthesis and subtraction techniques, supported by simultaneous light and radial velocity curve analysis to obtain accurate system parameters. Such well-focused, highresolution studies of a given RS CVn star not only contribute as another snapshot in its long-term activity behaviour, but also serve to improve our understanding of the atmospheric phenomenology in rapidly rotating cool stars.
Table 1. Phase ordered spectroscopic observation log of SV Cam.

\begin{tabular}{|c|c|c|c|c|}
\hline Date & $\begin{array}{l}\text { Exp. time } \\
\text { (s) }\end{array}$ & $\mathrm{HJD}_{\text {Mid }}$ & Phase $_{\text {Mid }}$ & SNR \\
\hline \multicolumn{5}{|c|}{ Calar Alto Observing run } \\
\hline 29.10 .2015 & 1500 & 57325.42262 & 0.143 & 61 \\
\hline 30.10 .2015 & 1500 & 57325.53672 & 0.324 & 78 \\
\hline 31.10 .2015 & 1500 & 57326.72813 & 0.333 & 64 \\
\hline 30.10 .2015 & 1500 & 57325.61552 & 0.457 & 71 \\
\hline 30.10 .2015 & 1500 & 57325.65956 & 0.531 & 79 \\
\hline 30.10 .2015 & 1500 & 57325.73145 & 0.652 & 91 \\
\hline 31.10 .2015 & 1500 & 57326.50529 & 0.945 & 84 \\
\hline \multicolumn{5}{|c|}{ La Palma Observing run } \\
\hline 17.01.2017 & 1800 & 57771.41699 & 0.144 & 97 \\
\hline 17.01.2017 & 1800 & 57771.49726 & 0.280 & 104 \\
\hline 15.01.2017 & 1800 & 57768.63556 & 0.455 & 81 \\
\hline 18.01.2017 & 1800 & 57771.61709 & 0.482 & 96 \\
\hline 16.01.2017 & 1800 & 57770.43934 & 0.496 & 83 \\
\hline 14.01.2017 & 1500 & 57767.53940 & 0.606 & 69 \\
\hline 18.01.2017 & 1800 & 57771.69724 & 0.617 & 99 \\
\hline 14.01.2017 & 1500 & 57767.55778 & 0.637 & 74 \\
\hline 17.01.2017 & 1800 & 57770.55080 & 0.684 & 91 \\
\hline 15.01.2017 & 1800 & 57769.38419 & 0.717 & 88 \\
\hline 18.01.2017 & 1800 & 57771.77563 & 0.749 & 102 \\
\hline 14.01.2017 & 1600 & 57767.64259 & 0.780 & 72 \\
\hline 14.01.2017 & 1600 & 57767.66169 & 0.813 & 74 \\
\hline 17.01.2017 & 1800 & 57770.64282 & 0.839 & 68 \\
\hline 15.01.2017 & 1800 & 57769.48775 & 0.892 & 71 \\
\hline 14.01.2017 & 1600 & 57767.73763 & 0.941 & 70 \\
\hline 17.01.2017 & 1800 & 57770.71976 & 0.969 & 71 \\
\hline
\end{tabular}

\section{OBSERVATIONS AND DATA REDUCTION}

The high-resolution time-series spectra of SV Cam were obtained between 2015 October 29 and 31 with the CAFE spectrograph (Aceituno et al. 2013) attached to the $2.2 \mathrm{~m}$ telescope at the Calar Alto Observatory (Almeria, Spain) and with the HERMES spectrograph (Raskin et al. 2011) attached to the $1.2 \mathrm{~m}$ Mercator telescope at the Roque de los Muchachos Observatory (La Palma, Spain) between 2017 January 12 and 18. At Calar Alto, we acquired seven CAFE spectra of the system that cover the wavelength range 4050$9095 \AA$ with an average resolution of $R=62000$. Each spectrum was acquired with an exposure time of $1500 \mathrm{~s}$ that gives signal-tonoise (SNR) values between $\sim 60$ and 90 . During the observing run in La Palma, we obtained 23 HERMES spectra of SV Cam with an average resolution of $R=85000$ and a wavelength coverage between 3780 and $9007 \AA$. Seventeen of these spectra have SNR between $\sim 70$ and 100. Six spectra were discarded as they have SNR $<40$ that makes them unsuitable for the subsequent analysis. The $\log$ of observations is given in Table 1 . The reduction of CAFE spectra that includes bias subtraction and flat field correction, removal of cosmic rays, wavelength calibration, and Heliocentric velocity correction was performed using the $\mathrm{IRAF}^{1}$ (Image Reduction and Analysis Facility) standard packages. The HERMES spectra were reduced with the automatic pipeline of the spectrograph (Raskin et al. 2011). Normalization of spectra was performed via a PYTHON

\footnotetext{
${ }^{1}$ IRAF is distributed by the National Optical Astronomy Observatory, which is operated by the Association of the Universities for Research in Astronomy, inc. (AURA) under cooperative agreement with the National Science Foundation.
} 

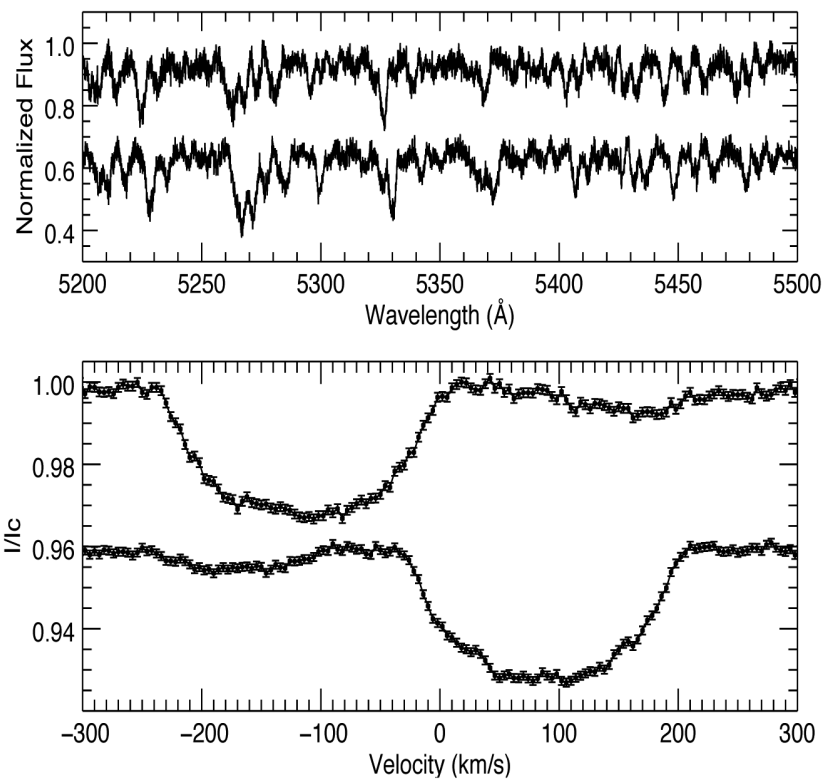

Figure 1. The top panel shows two input spectra taken at orbital phases 0.32 and 0.65 , while the lower panel shows the corresponding LSD profiles. Note that the strong and the weak dips in the LSD profiles belong to primary and secondary components, respectively.

code developed by our working group. This code can perform normalizations using polynomial and spline fits with the help of the synthetic spectra generated from model atmospheres. During our observing runs, three slowly rotating and non-active template stars, namely HD 143761 (G0V), HD 4628 (K2.5V), and HD 22049 (K2V), were also observed.

In order to determine better the spot signatures in the stellar spectra, we enhanced the signal using the LSD technique (Donati et al. 1997) and obtained mean velocity profiles with SNR values between 800 and 1100 for Calar Alto and La Palma data sets. The input lines list with line profile depths, which is required by the LSD technique, was extracted from the Vienna Atomic Line Database (Kupka et al. 1999), by considering the $\log g$ and $T_{\text {eff }}$, appropriate for SV Cam. During the preparation of the line list, wavelength regions covering lines affected by chromospheric heating (e.g. Ca II $\mathrm{H} \& \mathrm{~K}, \mathrm{H} \alpha$, and $\mathrm{Na} \mathrm{D}$ ) and strong telluric lines were discarded in order to prevent artefacts in the LSD profiles. In addition to SV Cam, the LSD profiles of the template stars were also computed and subsequently used for the generation of the lookup tables (used to model the local intensity profile). We show a sample of input spectra from Calar Alto observations and their corresponding LSD profiles in Fig.1. Time-series plots of the LSD profiles showing the data coverage as well as the radial velocity variations are shown in Figs 2 and 3 for the Calar Alto and La Palma data sets, respectively. The radial velocity variation of the secondary component is barely visible due to the weak contribution of that component to the total flux.

High precision $B, V, R, I$, and Strömgren $b$ and $y$ photometry of SV Cam were obtained using the iKon L 936 Andor CCD camera attached to the $50 \mathrm{~cm}$ Cassegrain telescope at the Astronomical Observatory of the Jagiellonian University during two nights (2015 December 31 and 2016 January 1). We obtained 789, 721, 1488, 2116, 776, and 771 individual measurements in $B, V, R, I, b$, and $y$ bands, respectively. We chose GSC $4538-723$ (K0V) as the comparison star to derive the differential magnitudes. We also observed

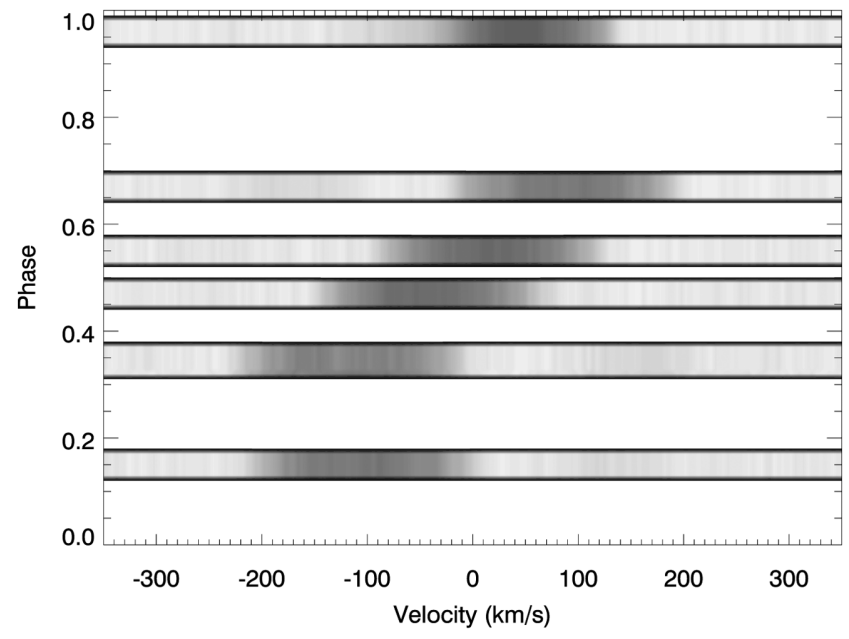

Figure 2. A grey-scale image representation of Calar Alto data set obtained using the LSD profiles of SV Cam.

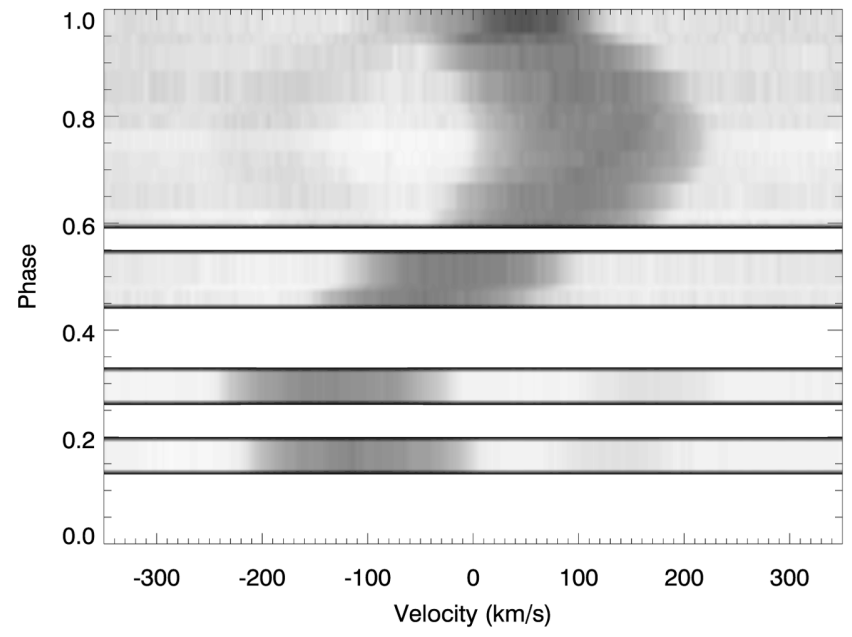

Figure 3. Same as Fig. 2 but for La Palma data set.

TYC 4538-705-1 ( $B-V=0.84)$ as check star, in order to check the variability of the comparison. The reduction procedure concerning the correction of scientific images for bias, dark, and flat-field was performed with IRAF while the C-MUNIPACK software (Hroch 1998), an interface for the DАОРНОТ package, was applied for derivation of magnitudes.

\section{ORBITAL SOLUTION AND PHOTOMETRIC ANALYSIS}

Eclipsing binary stars provide a unique opportunity to obtain the stellar masses directly as well as the orbital parameters, when both photometric and spectroscopic data are available. We first determined the radial velocities (RV) for each component of the system using both Calar Alto and La Palma spectra. As a first attempt, we used the IDL routines based on the broadening functions method improved by Rucinski (1999). However, since the flux contribution of the secondary component to the total flux of the system is very small, we could not estimate precisely the RVs of the secondary component. The LSD technique, on the other hand, is a powerful tool that uses thousands of photospheric lines to enhance the SNR of the spectra. Consequently, we determined the radial velocities of 


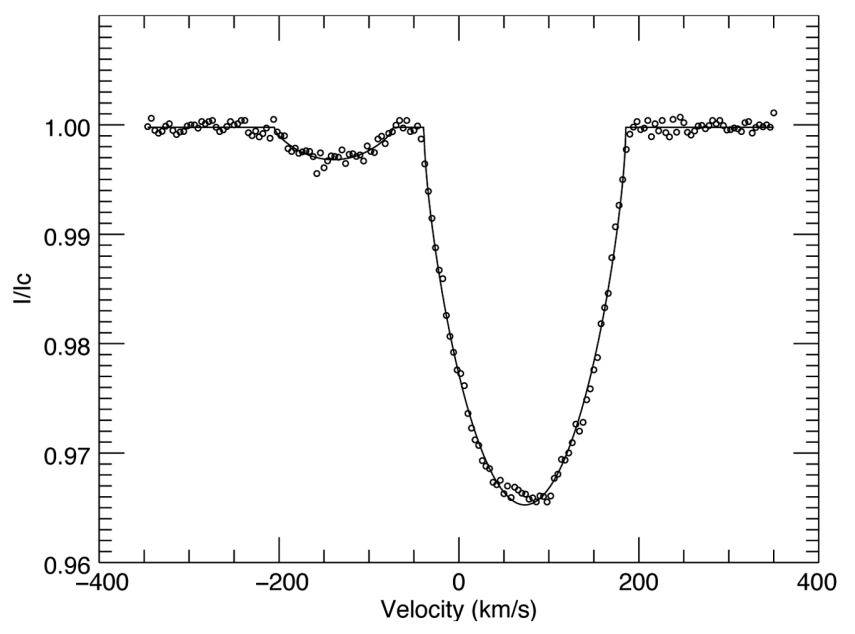

Figure 4. An example of LSD profile (open circles) at phase $=0.60 \mathrm{ob}-$ tained from La Palma observations and the synthetic LSD fit (solid line) used in radial velocity determination.

both components of SV Cam by fitting synthetic rotation profiles to the LSD profiles with the help of the equation (equation 1) given by Barnes et al. (2004). This equation is the improved version of the form (equations 17-12) given by Gray (1992), which is based on the determination of a rotationally broadened profile via the convolution of a non-rotating star profile and a rotation profile by considering the limb darkening effect. The modified version of this equation given by Barnes et al. (2004) additionally enables the adjustment of continuum level, width, depth, and the radial velocities of each profile. An example of a fitted profile obtained in the radial velocity determination process is presented in Fig. 4. We applied the related technique individually for both spectroscopic data sets.

The simultaneous light curve and radial velocity analysis of SV Cam was performed with the PHOEBE (Prša \& Zwitter 2005) code. We assumed a detached system configuration and carried out computations simultaneously for the RV data from La Palma and Calar Alto observations. We set the gravity darkening and the albedo coefficients to 0.32 (Lucy 1967) and 0.5 (Ruciński 1969), respectively, which are theoretical values for a convective envelope appropriate for the spectral types of both components. The limb darkening coefficients were interpolated automatically from the van Hamme (1993) tables under the linear-cosine law assumption. We fitted the orbital inclination $(i)$, effective temperature of the secondary component $\left(T_{2}\right)$, surface potentials of components $\left(\Omega_{1,2}\right)$, the luminosity of the primary component $\left(L_{1}\right)$, the system mass ratio $(q=$ $\left.M_{2} / M_{1}\right)$, semimajor axis $(a)$, and the gamma velocity $\left(V_{\gamma}\right)$. There are several studies in the literature concerning the effective temperature of the primary component of SV Cam system ranging from $5700 \mathrm{~K}$ (Patkos \& Hempelmann 1994) to $6440 \mathrm{~K}$ (Albayrak et al. 2001). Jeffers et al. (2006b) used the spectrophotometric HST data and determined the primary effective temperature as $6039 \pm 58 \mathrm{~K}$. Consequently, we fixed the primary temperature to that value in our computations.

The light and radial velocity curve analysis of SV Cam showed that convincing solutions can only be achieved via cool spot modelling. Most of the photometry-based spot modelling studies published in the literature mentioned the presence of one or two cool spots with their locations and sizes being variable from one season to another. Therefore, we performed the spot modelling by assuming one, two, and even three cool spots located on the surface of the primary component and adjust all the spot-related parameters
Table 2. Results from the simultaneous light and radial velocity curve analysis of SV Cam.

\begin{tabular}{lcc}
\hline Stellar parameters & $\begin{array}{c}\text { Solution 1 } \\
\text { (La Palma RVs) }\end{array}$ & $\begin{array}{c}\text { Solution 2 } \\
\text { (Calar Alto RVs) }\end{array}$ \\
\hline$T_{1}[\mathrm{~K}]$ & 6039 & 6039 \\
$T_{2}[\mathrm{~K}]$ & $4356(7)$ & $4364(10)$ \\
$i\left[^{\circ}\right]$ & $87.26(13)$ & $87.24(17)$ \\
$q=M_{2} / M_{1}$ & $0.641(2)$ & $0.618(3)$ \\
$K_{1}\left[\mathrm{~km} \mathrm{~s}^{-1}\right]$ & $124.9(1.6)$ & $121.2(2.9)$ \\
$K_{2}\left[\mathrm{~km} \mathrm{~s}^{-1}\right]$ & $188.6(1.9)$ & $196.1(3.3)$ \\
$\Omega_{1}$ & $3.58(1)$ & $3.54(1)$ \\
$\Omega_{2}$ & $4.19(2)$ & $4.08(2)$ \\
$a\left[\mathrm{R}_{\odot}\right]$ & $3.72(1)$ & $3.75(1)$ \\
$V_{\gamma}\left[\mathrm{km} \mathrm{s}^{-1}\right]$ & $-11.39(20)$ & $-10.37(28)$ \\
$L_{1} /\left(L_{1}+L_{2}\right)[B]$ & $0.967(6)$ & $0.966(6)$ \\
$L_{1} /\left(L_{1}+L_{2}\right)[V]$ & $0.945(7)$ & $0.943(7)$ \\
$L_{1} /\left(L_{1}+L_{2}\right)[R]$ & $0.924(6)$ & $0.922(7)$ \\
$L_{1} /\left(L_{1}+L_{2}\right)[I]$ & $0.906(7)$ & $0.904(7)$ \\
$L_{1} /\left(L_{1}+L_{2}\right)[b]$ & $0.956(7)$ & $0.955(7)$ \\
$L_{1} /\left(L_{1}+L_{2}\right)[y]$ & $0.943(7)$ & $0.941(7)$ \\
$S p t$ &
\end{tabular}

Spot parameters (on primary component)

$\begin{array}{lc}\varphi_{1}\left[^{\circ}\right] & 41.3(1.2) \\ \lambda_{1}\left[{ }^{\circ}\right] & 294.8(1.1) \\ \theta_{1}\left[^{\circ}\right] & 30.8(9) \\ T F_{1} & 0.89(6) \\ \varphi_{2}\left[^{\circ}\right] & 42.2(1.4) \\ \lambda_{2}\left[^{\circ}\right] & 45.1(9) \\ \theta_{2}\left[^{\circ}\right] & 30.1(1.2) \\ T F_{2} & 0.90(7)\end{array}$

Absolute parameters

$\begin{array}{lcc}M_{1}\left[\mathrm{M}_{\odot}\right] & 1.19(1) & 1.24(1) \\ M_{2}\left[\mathrm{M}_{\odot}\right] & 0.76(2) & 0.77(2) \\ L_{1}[\mathrm{~L} \odot] & 1.99(2) & 2.04(2) \\ L_{2}[\mathrm{~L} \odot] & 0.21(2) & 0.24(2) \\ R_{1}\left[\mathrm{R}_{\odot}\right] & 1.31(1) & 1.30(2) \\ R_{2}\left[\mathrm{R}_{\odot}\right] & 0.81(2) & 0.81(2) \\ R_{1}-\text { pole }\left[\mathrm{R}_{\odot}\right] & 1.25(1) & 1.27(2) \\ \mathrm{R}_{2}-\text { pole }\left[\mathrm{R}_{\odot}\right] & 0.79(2) & 0.80(2) \\ \log g_{1}[\mathrm{cgs}] & 4.29(3) & 4.30(3) \\ \log g_{2}[\mathrm{cgs}] & 4.52(3) & 4.51(3) \\ \Sigma(O-C)^{2}[B] & 0.048 & 0.047 \\ \Sigma(O-C)^{2}[V] & 0.045 & 0.045 \\ \Sigma(O-C)^{2}[R] & 0.110 & 0.109 \\ \Sigma(O-C)^{2}[I] & 0.250 & 0.251 \\ \Sigma(O-C)^{2}[b] & 0.061 & 0.060 \\ \Sigma(O-C)^{2}[y] & 0.055 & 0.055\end{array}$

$\varphi_{i}-$ colatitude of spot, $\lambda_{i}$ - longitude of spot, $\theta_{i}$ - angular radius of spot, $T F_{i}$ - temperature factor of spot. Formal errors from the PHOEBE code were given in parentheses. Errors of spot parameters were obtained during the solution using La Palma RVs.

(colatitude, longitude, radius, and temperature factor) during the iterations. We could not obtain a reliable model under a single cool spot assumption, since the light-curve asymmetries throughout the orbital phases show distinctive amounts of intensity drops, which is likely a consequence of different spot temperatures and/or radii. In the case of three cool spots, the solutions did not converge unless the temperature of the third spot would be equal to the photospheric temperature of the primary component. Consequently, we found a converged solution under the assumption of two cool spots on the primary component. The results we derived from this analysis are presented in Table 2, while the model light curves obtained using 


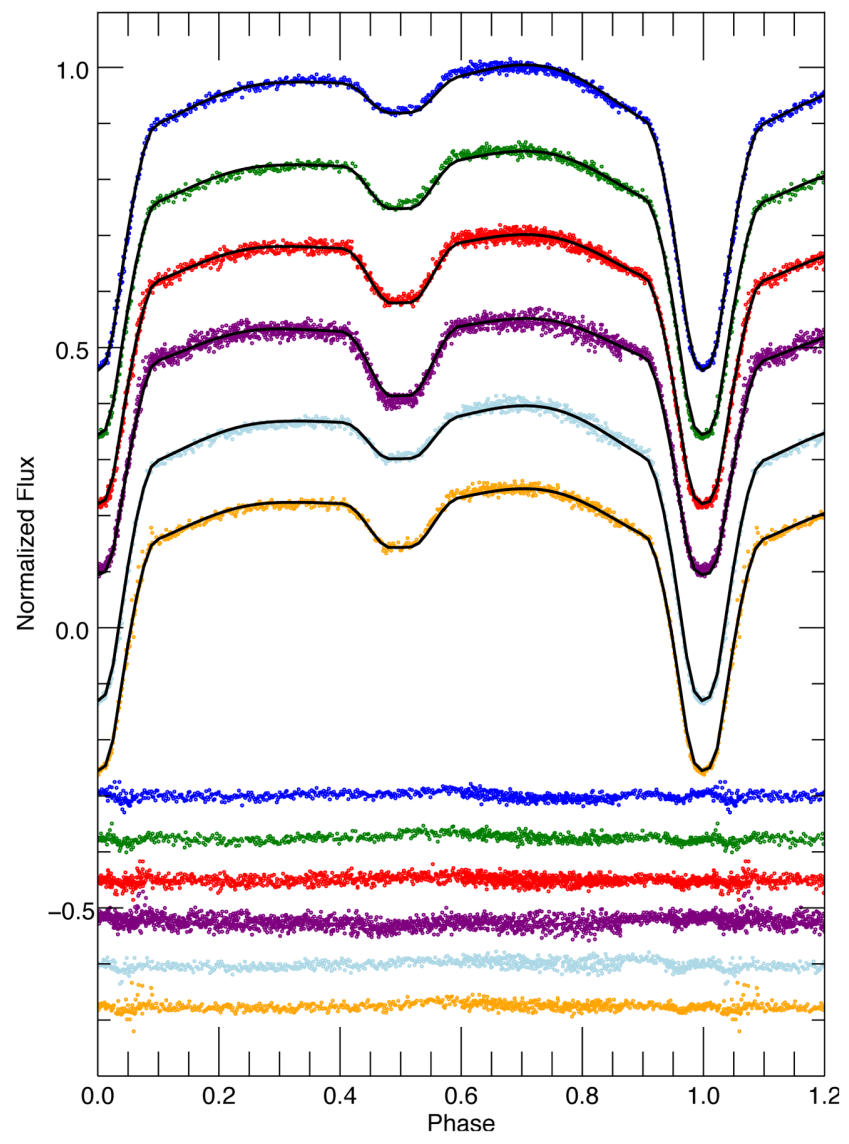

Figure 5. BVRIby light curves of SV Cam. Blue, green, red, purple, light blue, and orange dots represent the observed light curves in the $B, V, R, I, b$, and $y$ filters, respectively, while the solid lines show best fits obtained from the simultaneous analysis using La Palma RV curves and the multiband photometry. Except the ones related with $B$ band, all the observed and synthetic light curves are shifted arbitrarily for clarity by 0.15 , in relative flux units, and the residuals by 0.075 .

the RV data from La Palma and the radial velocity curves from both data sets are shown in Figs 5, 6, and 7, respectively. The mass ratio (q) obtained using the HERMES data from the simultaneous light curve and radial velocity data analysis is identical to that determined by Rucinski et al. (2002), as well as the masses of both components, which are also consistent. The other absolute parameters (i.e. $R_{1,2}$, $L_{1,2}$ ) and the parameters related to system geometry (i.e. $\Omega_{1,2}, i$ ) are also in accordance with those of given in the literature.

\section{DOPPLER IMAGING}

In order to map the primary component of SV Cam, we used the Doppler imaging code DOTs (Collier Cameron 1997), which, based on the maximum entropy method (MEM), finds the best-fitting spot distribution across the stellar surface. The code can also be used to determine the system parameters accurately, by performing maximum entropy regularized iterations to find which set of parameters yields the best fit to the line profiles. The parameter optimization is very critical in that sense, as the inaccurate determination of system parameters can lead to artefacts in the surface maps (e.g. Unruh 1996). Moreover, compared to single stars, Doppler imaging of bi-

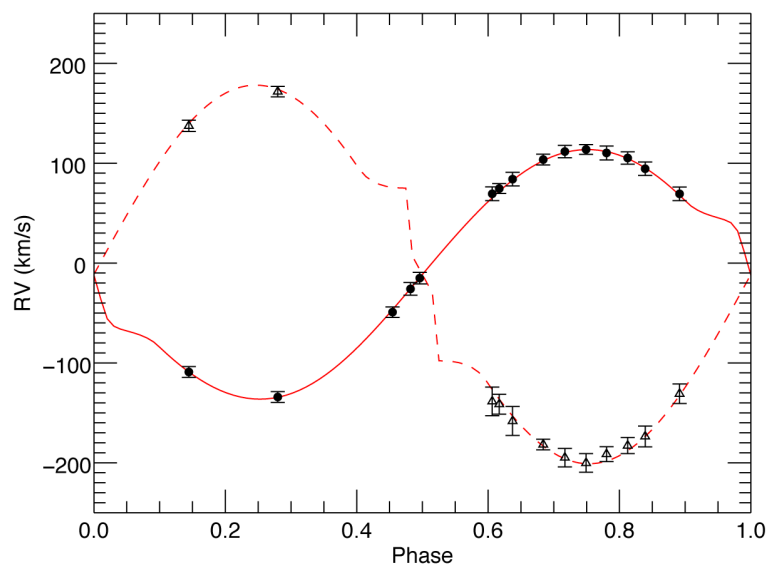

Figure 6. Radial velocity curve of SV Cam obtained using HERMES spectra. The solid and dashed red lines represent the RV fit to the data, while the filled circles and open triangles belong to the radial velocity data of the primary and secondary components, respectively.

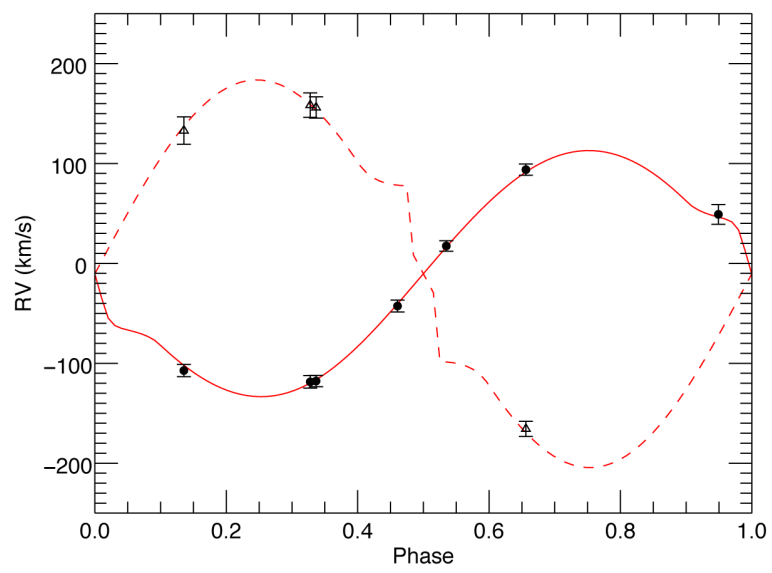

Figure 7. Same as Fig. 6 but for the CAFE (Calar Alto) data set.

nary systems requires many more parameters, making the accurate system parameter determination crucial.

Based on the two-temperature model to represent photosphere(s) and $\operatorname{spot}(\mathrm{s})$, DOTs needs lookup tables generated using the spectra of template stars to calculate the intensity contributions of each element across the stellar surface. In this context, the spectra of HD 143761 (G0V) and HD 4628 (K2.5V), obtained during both Calar Alto and La Palma observing runs, are used to represent the photospheres of the primary and secondary components. Assuming the spot temperature to be $1000 \mathrm{~K}$ lower $(\sim 5000 \mathrm{~K})$ than the photosphere of the primary component, as follows from the studies by Zboril \& Djuraševič (2004) and Hempelmann et al. (1997), we used the spectra of HD 22049 (K2V) from both runs to represent the spectrum of the star-spot(s). We determined the linear limb darkening coefficients using the tables by van Hamme (1993), taking into account the effective temperatures of both components.

Under the assumption of two cool spots, the simultaneous analysis of high-quality BVRIby light curves together with the radial velocities enabled us to estimate the system parameters accurately. During the surface reconstruction via DOTs, we used the set of parameters obtained from simultaneous $\mathrm{LC}+\mathrm{RV}$ solutions from $\mathrm{La}$ Palma data set. These RV measurements are more numerous and therefore the obtained results are more reliable, especially for the 


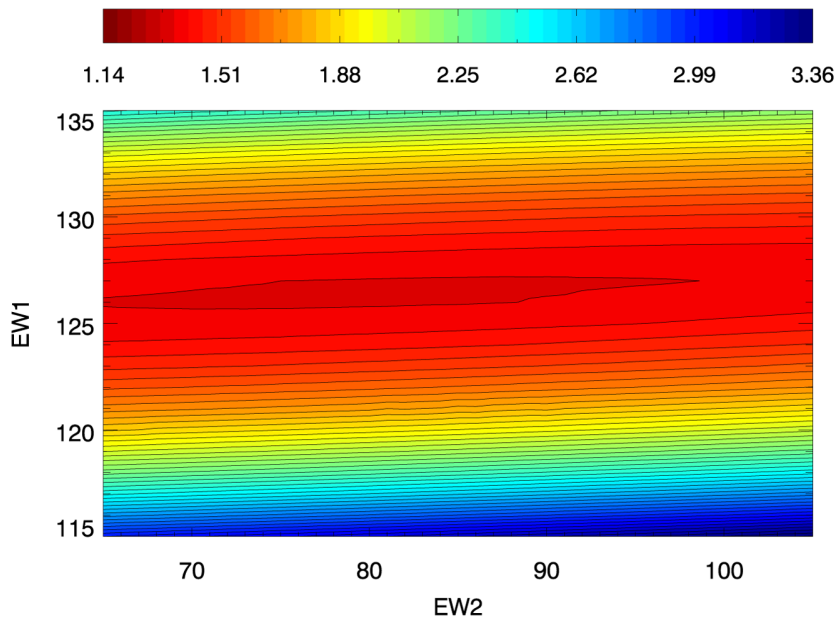

Figure 8. Two-dimensional grid search for the optimization of $E W_{1,2}$ parameters.

system orbital parameters. As can be seen from Table 2, centre-ofmass velocities $\left(V_{\gamma}\right)$ reckoned by the solutions of the two RV curves obtained with HERMES and CAFE spectrographs more than one year apart from each other have $\sim 1 \mathrm{~km} \mathrm{~s}^{-1}$ difference. In addition, the orbital period variation of the system shows a cyclic variation that is attributed to the existence of an additional body, in the literature. This $V_{\gamma}$ variation could be due to a third body, but we cannot exclude a systematic effect related to the different instruments. However, this difference in $V_{\gamma}$ was taken into account during the surface reconstruction performed for both data sets. PHOEBE gives the arithmetic mean of $r_{\text {pole }}, r_{\text {side }}$, and $r_{\text {back }}$ parameters, whereas DOTs requires the polar radii as the input parameter. The errors in Table 2 are the formal errors given by the PHOEBE code.

The $E W$ parameter in the DOTs code controls the strength of the synthetic LSD lines that are generated using the spectra of template stars, which represent the local intensity profiles of the photosphere(s) and spot(s). This is adjusted to optimally fit the entire data set as part of the parameter optimization process. Therefore, the accurate determination of line $E W_{1,2}$ parameters are also critical, as it affects both the widths and depths of the modelled line profiles. This is particularly important in preventing the artificial high-latitude spots during the surface reconstruction. In order to determine the optimum values of $E W_{1,2}$, we carried out a two-dimensional grid search separately for both data sets, using DOTs for the optimization of $E W_{1,2}$. The resulting values of $E W_{1,2}$ optimization for the Calar Alto data set are shown in Fig. 8. During surface reconstructions, we used the TEST statistics option of DOTs to determine the degree of convergence for maximum entropy as the stopping criteria (see Şenavc1 et al. 2011 for details). The data sets and the best fit models are shown in Figs 9 and 11, while the corresponding maps are in Figs 10 and 12, respectively. Both sets of maps show a symmetric distribution of spots, which can be attributed to the inability of DI techniques to determine on which the hemisphere the $\operatorname{spot}(\mathrm{s})$ are located at, in systems with inclination angles approaching $90^{\circ}$.

\section{PHOTOMETRIC MAPPING}

Prior to mapping our photometric light curves using DOTs, we must first simulate the unspotted light curve. To do this, we carried out the following steps. We chose the $R$-band light curve of the system, since the central wavelength of our spectral range is close to that band. We then generated an unspotted light curve with DOTs, using

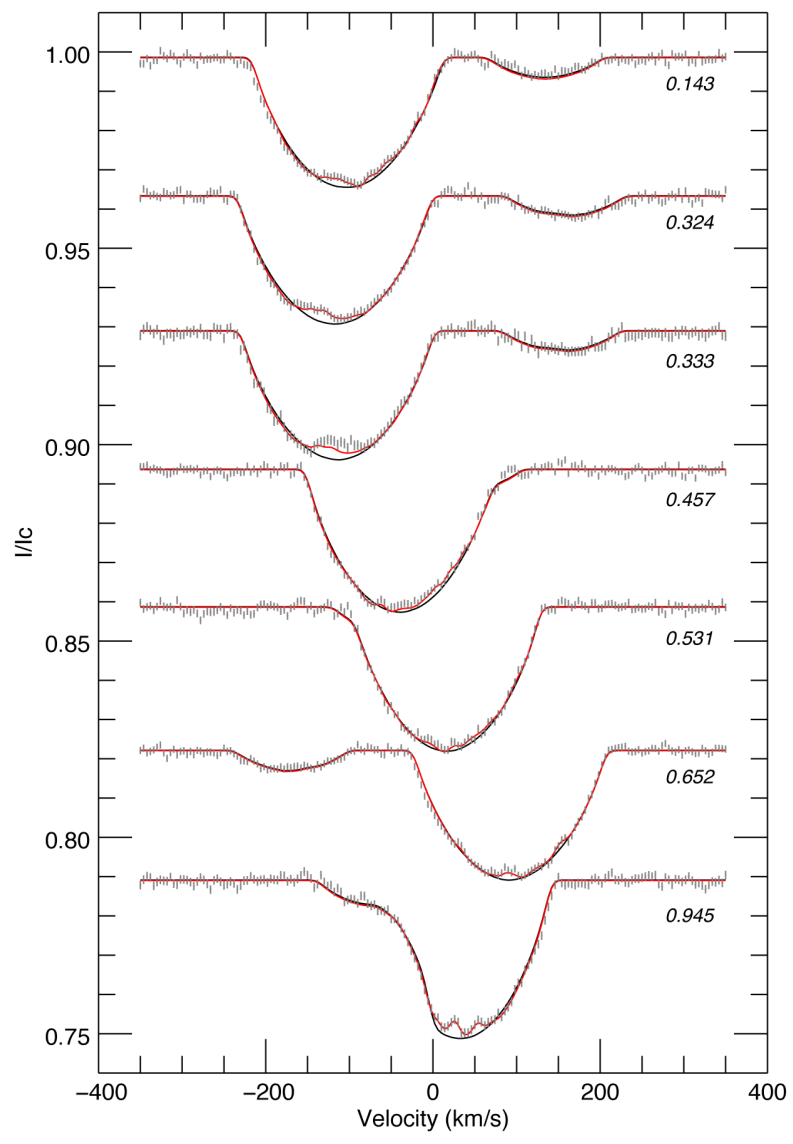

Figure 9. Phase-ordered LSD profiles together with the error bars for Calar Alto data set. Black solid lines represent the synthetic velocity profiles generated using the system parameters, while the red solid lines show the maximum entropy regularized models of SV Cam.

exactly the same parameters from PHOEBE solution and local intensity models based on the PHOENIX models. A comparison of unspotted synthetic light curves generated using both DOTS and PHOEBE codes is shown in Fig. 13. The $\Sigma(O-C)^{2}$ value of 0.0025 obtained from that comparison clearly shows that the output from both codes is in agreement.

After testing the consistency of unspotted system configuration, we carried out the photometric mapping for the primary component of SV Cam. The best fit to the $R$-band light curve of the system and the resulting map for the primary component are plotted in Figs 14 and 15, respectively. Further tests and a discussion of the map will be given in Section 6.1.4.

\section{RESULTS AND DISCUSSION}

We have investigated the surface inhomogeneities of the primary component of the RS CVn type eclipsing binary SV Cam via the code DOTs, using high-resolution time-series spectra obtained from Calar Alto and La Palma observatories. The high-quality multiband photometric data, taken in two consecutive nights, also allowed us to determine the system parameters accurately, with the help of the light and radial velocity curve analysis performed simultaneously. We also obtained the surface map of the primary component using the $R$-band light curve of the system, this time using the photometric mapping mode of DOTs. The Calar Alto data include only seven time-series spectra mostly distributed between orbital phases 0.0 

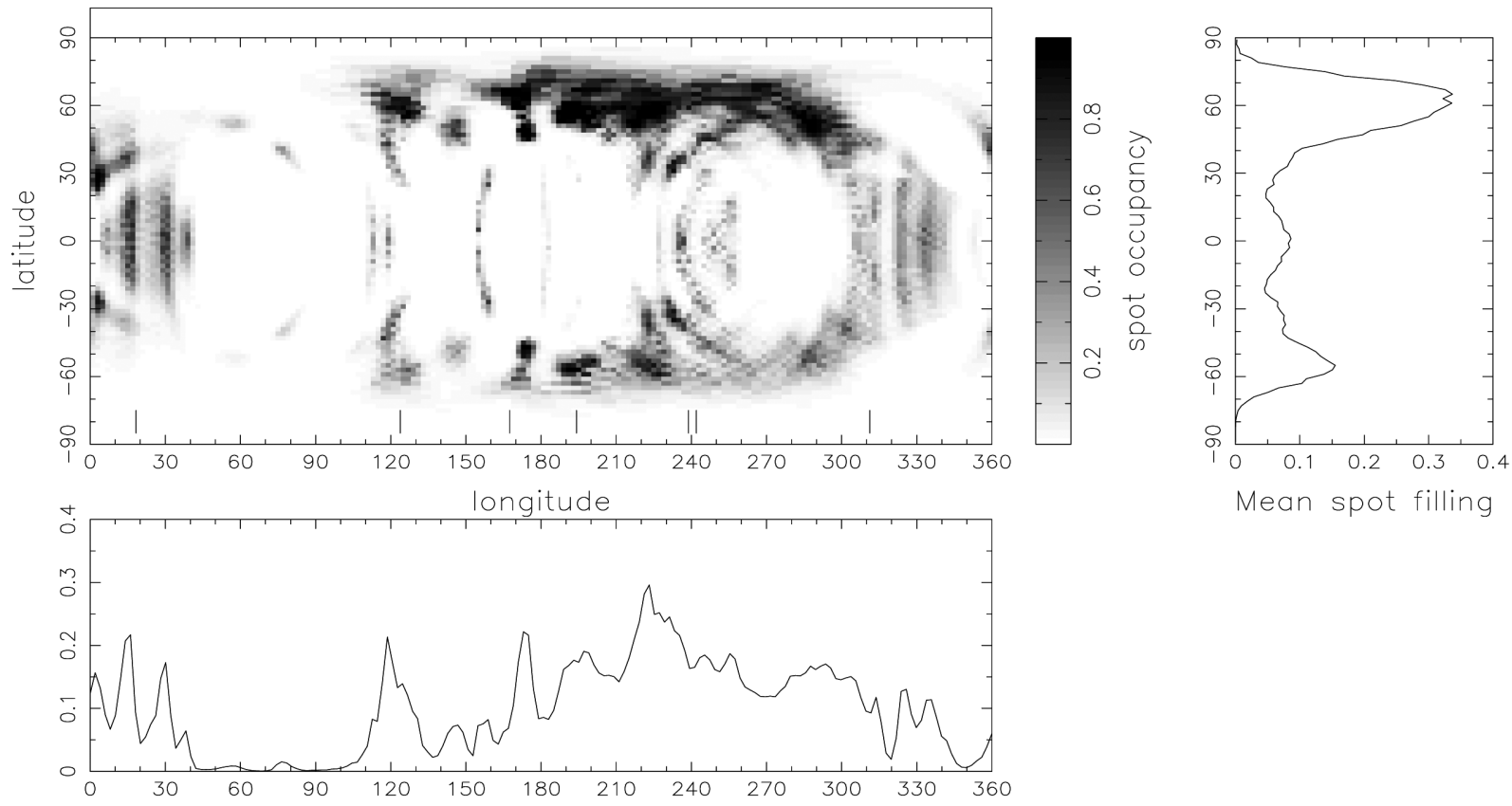

Figure 10. Mercator projection of reconstructed image for the primary component of SV Cam obtained using the Calar Alto data set that are acquired between 2015 October 19 and 31. Right-hand panel shows the latitudinal spot filling factor, while the lower left panel shows the longitudinal correspondence.
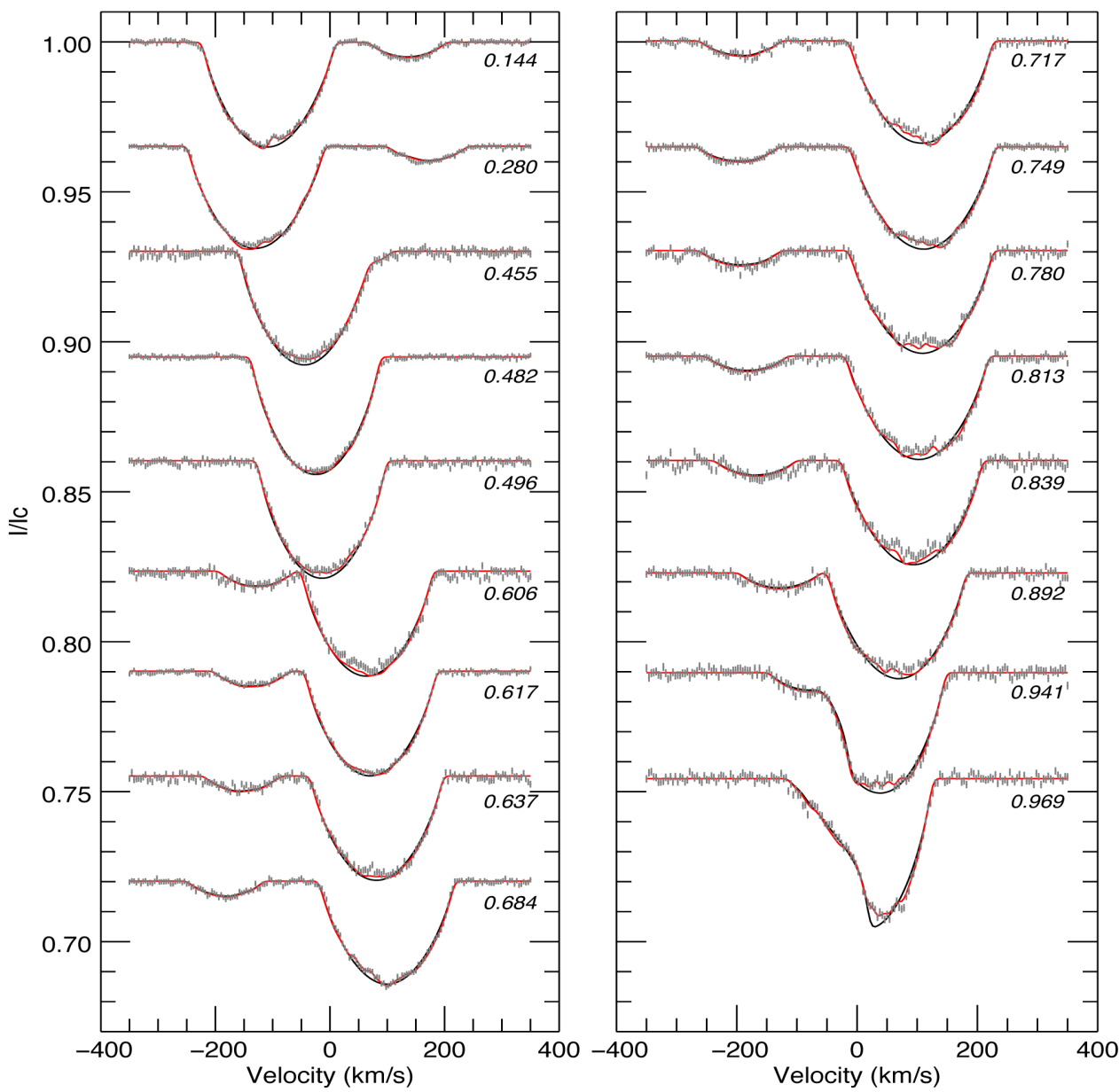

Figure 11. Phase-ordered spectra for the La Palma data set. Error bars and synthetic profiles are plotted as for the Calar Alto data in Fig. 9. 

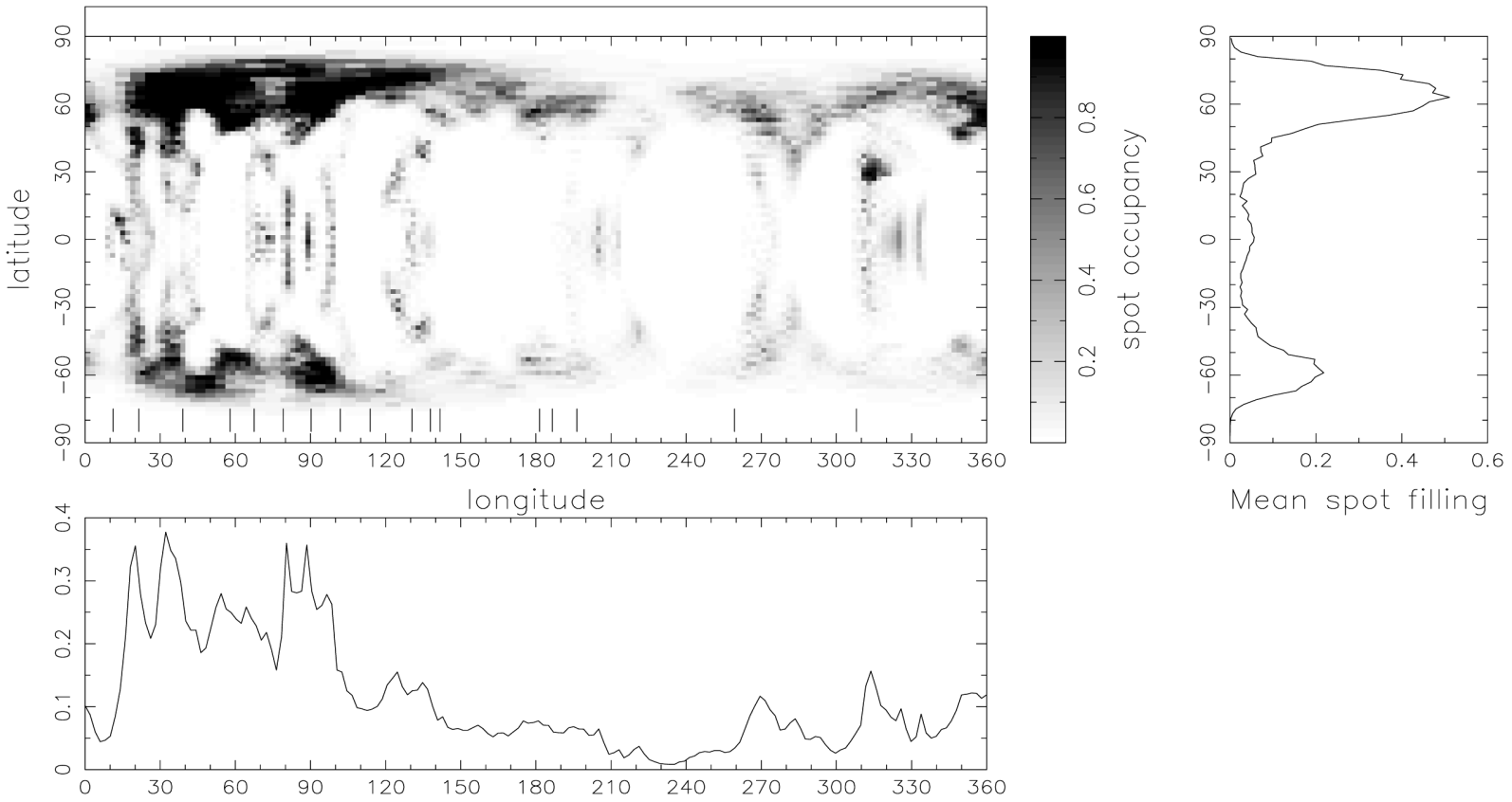

Figure 12. Same as Fig. 10 but for La Palma data set obtained between 2017 January 12 and 18.

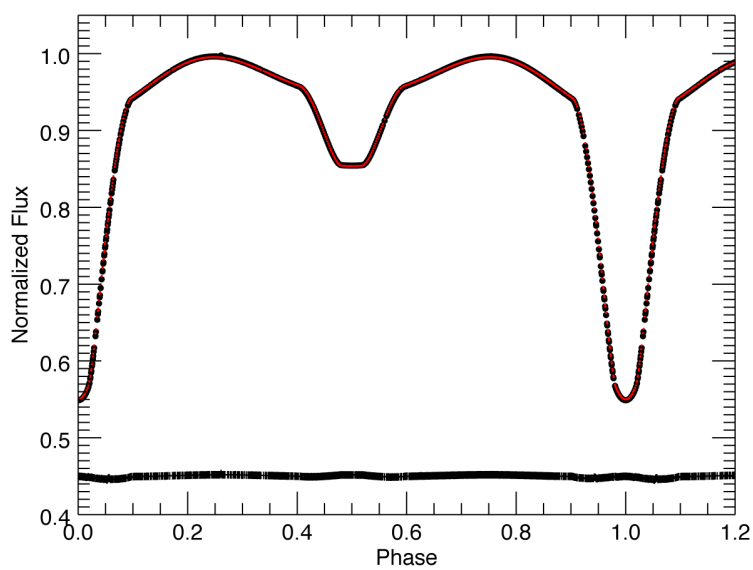

Figure 13. Comparison of unspotted $R$-band light curves of SV Cam obtained using DOTs (black circles) and PHOEBE (red circles) together with the residuals.

0.5 that corresponds to the longitudes $360^{\circ}$ to $180^{\circ}$ (note that phase runs in reverse to longitude). The La Palma data including 17 timeseries spectra on the other hand have better phase coverage mainly concentrated between longitudes $0^{\circ}$ and $200^{\circ}$. However, we have only two spectra distributed between longitudes $200^{\circ}$ and $360^{\circ}$. The tests showed that surface reconstructions with such poor data sampling, especially like the Calar Alto data in our case, mostly carry reliable information about the distribution of large-scale spots and show smearing or absence of small-scale star-spot features in the surface maps (Xiang et al. 2015).

\subsection{Star-spot distributions}

\subsubsection{Doppler images}

The surface map obtained using Calar Alto data (Fig. 10) shows high-latitude spot features extended to lower latitudes and predomi-

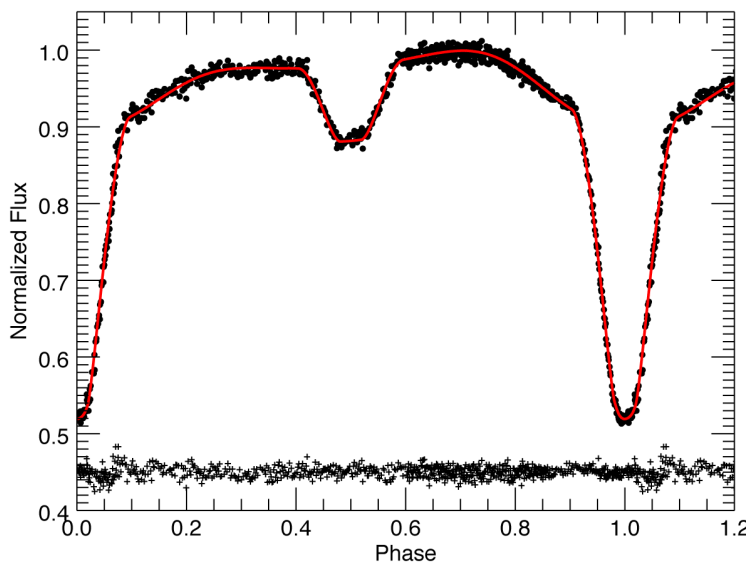

Figure 14. The best fit (red solid lines) and residuals (plus signs) for the $R$-band data of SV Cam obtained using the photometric mapping mode of the code Dots. Here, the $\Sigma(O-C)^{2}$ is 0.058 . The resultant map (Fig. 15) shows two predominant spot features centred at around longitudes $\sim 50^{\circ}$ and $300^{\circ}$.

nantly located between longitudes $150^{\circ}$ and $300^{\circ}$, with a maximum longitudinal spot filling factor value of $\sim 0.3$. There is a spotless zone between longitudes $\sim 40^{\circ}$ and $100^{\circ}$, due to the deficiency and uneven distribution of data throughout the orbital cycle. The surface map from La Palma data (Fig. 12) shows predominant high-latitude spot features between longitudes $0^{\circ}$ and $150^{\circ}$ with a slightly higher filling factor than that of the map obtained using Calar Alto data. It is not possible to make interpretations on the migration of spot features, due to the inhomogeneous distribution of spectral data and the large interval between the two observing runs ( $\sim 14$ months). What is clear is that there is heavy spot coverage where there is good phase coverage in both maps. This can represent the actual longitudinal distribution of spots, which is similar to the those obtained by Hempelmann et al. (1997), who also used a MEM-based DI code, but with more evenly sampled phases throughout the orbital 

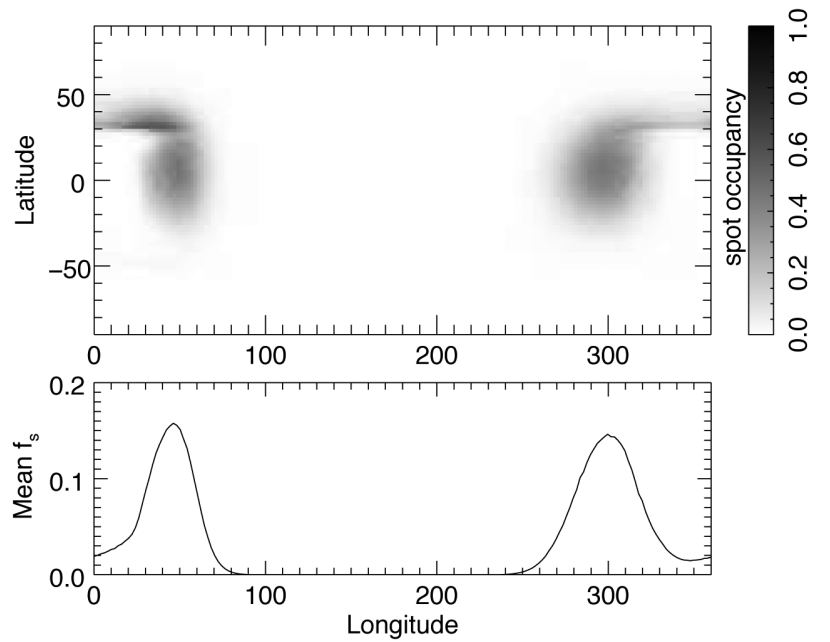

Figure 15. Mercator projection (upper panel) and longitudinal spot filling factor distribution (lower panel) of reconstructed image for the primary component of SV Cam obtained using the $R$-band data of the system acquired between 2015 December 31 and 2016 January 1. Note that no latitude information can be obtained from photometry using only one band.

period. Alternatively, the actual distribution can be more homogeneous (axisymmetric), and the longitudinal profile we obtained was thus affected by the phase sampling.

The overall distribution of high-latitude spot features, as well as the extensions to the lower latitudes are clear from our maps. This is expected in such active rapid rotators, due to the enhanced Coriolis force acting on rising flux tubes (Schuessler \& Solanki 1992; Holzwarth \& Schüssler 2003; Iş1k, Schmitt \& Schüssler 2011). Jeffers et al. (2005) revealed the existence of a polar spot on the primary component using precise spectrophotometric HST data. The presence of a polar spot on the surface of an RS CVn type binary $\zeta$ was directly imaged by Roettenbacher et al. (2016), using long-baseline infrared interferometry. Besides, most of the RS CVn type binaries are known to have polar caps (Strassmeier 2009). However, most of such systems have lower orbital inclinations than that of SV Cam. We will therefore test the reliability of the latitudinal distributions of our maps, which do not show polar spots, in Section 6.1.3.

\subsubsection{Numerical tests on phase smearing}

The CAFE data of SV Cam was obtained using $1500 \mathrm{~s}$ of exposure time, while 1500,1600 , and 1800 s of exposure times were used during the HERMES observing run. These exposure times correspond to $2.9,3.1$, and 3.5 per cent of the orbital period of SV Cam, respectively, which cause the well-known phase smearing phenomenon. The smearing produced by the maximum exposure time of 1800 $\mathrm{s}$ is about 0.035 in units of the orbital period, $\sim 12^{\circ}$ in longitude, or $\sim 15 \mathrm{~km} \mathrm{~s}^{-1}$ in radial velocity. It causes the blurring of the LSD profiles and hence affects the resultant maps, preventing the reconstruction of smaller surface features that can be resolved via shorter exposure times. It is possible to take into account the phase smearing effect within the DOTs code, under a reasonable number of profiles as well as the number of data points for each profile, depending on the available computational power and memory limitations. We have not carried out a direct high-performance reconstruction of the Doppler images by considering the exposure times. Instead, we have tested the effects of smearing, by generating artificial profiles resulting from five spots on the primary component with different
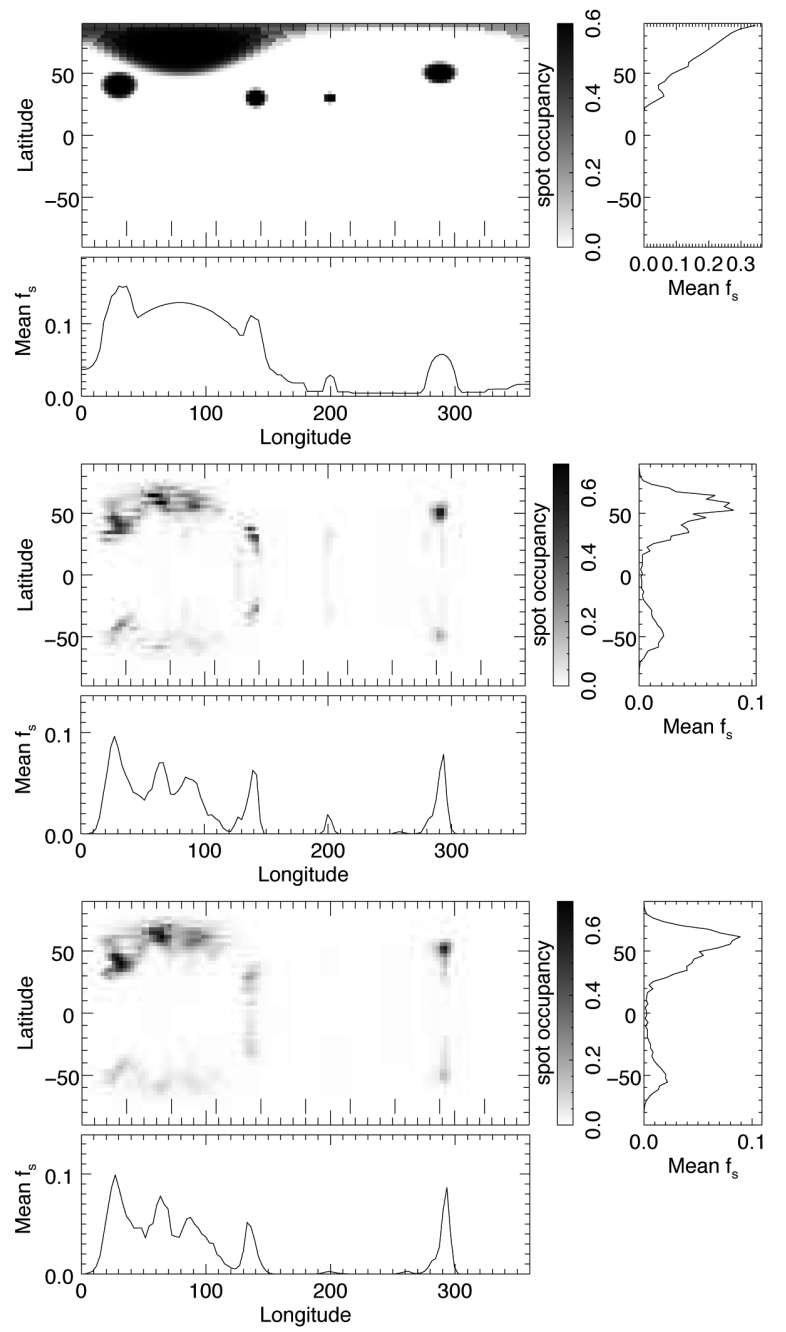

Figure 16. Reconstructions for the input spot map (upper panel); S1.0 (middle panel) and S3.5 (lower panel).

locations and sizes (between $4^{\circ}$ and $20^{\circ}$ ), using the same system parameters of SV Cam. The artificial LSD profiles were generated with 0.1 phase intervals. To mimic the smearing levels of 1 and 3.5 per cent of the orbital period, the artificial data were produced using 500 and $1800 \mathrm{~s}$ of exposure times, respectively. We call these two cases S1.0, corresponding to the conventional theoretical limit to avoid any measurable effects of smearing, and S3.5, to represent our longest exposure time. The resulting reconstructions are shown in Fig. 16.

The highest latitude spot on both S1.0 and S3.5 maps is reconstructed at lower latitudes, as a consequence of high orbital inclination of the system, which is discussed in Section 6.1.3, in detail. The reconstructed spots on the S3.5 map are blurred compared to those on $\mathrm{S} 1.0$. The smallest spot, which has a radius of $4^{\circ}$ and located at $200^{\circ}$ longitude, is resolved on S1.0 and disappears on S3.5. Therefore, our exposure times do not lead to considerable uncertainties for relatively large spots in Figs 10 and 12, whereas features with sizes smaller than about $12^{\circ}$ (corresponding to our longest exposure time) can be artefacts of the inversion procedure. 

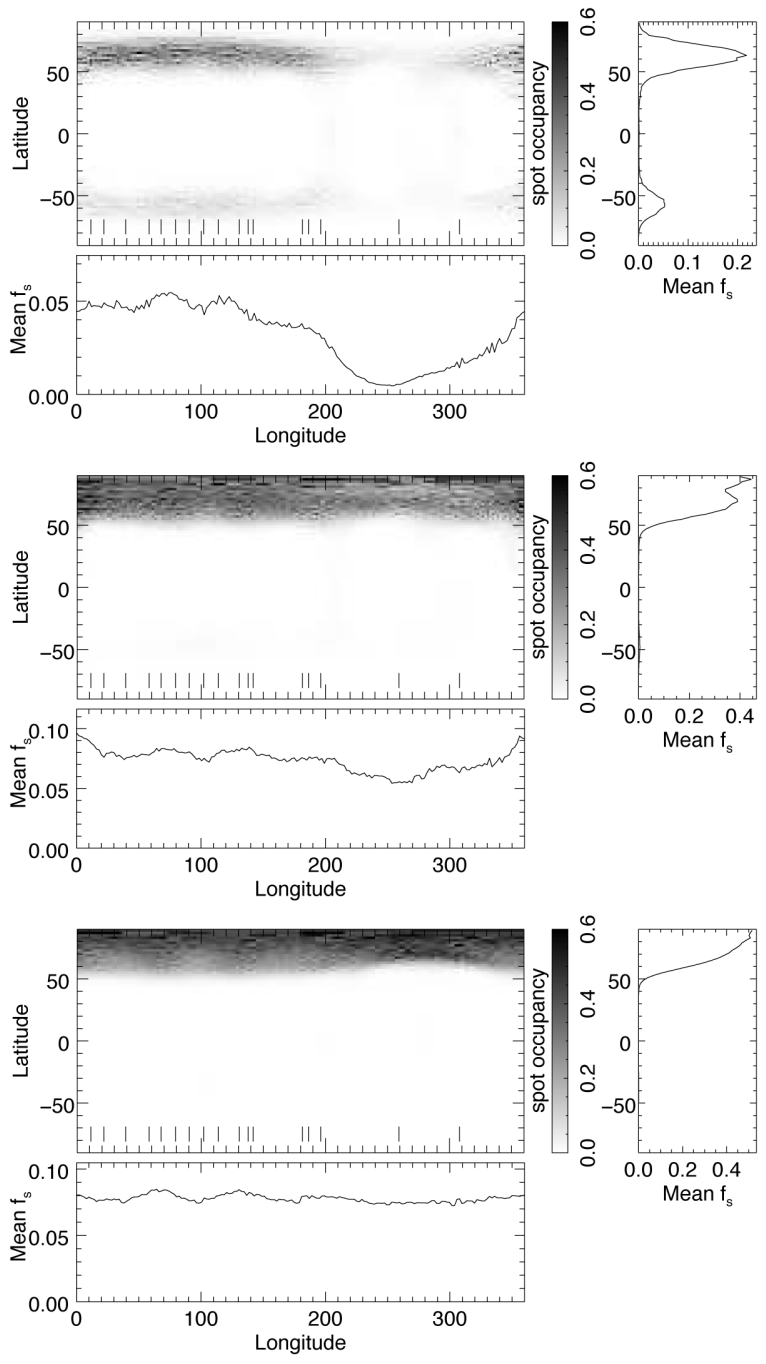

Figure 17. Test results concerning the reconstruction of a polar spot under the orbital inclinations $i=87.2^{\circ}$ (top panel), $80^{\circ}$ (middle panel), and $70^{\circ}$ (bottom panel).

\subsubsection{Numerical tests on the orbital inclination}

We performed simulations in order to test the ability of our surface reconstructions to recover polar spots at different inclination angles approaching $90^{\circ}$. In this context, using exactly the same system parameters of SV Cam, except for the orbital inclination, we simulated a polar spot with a radius of $30^{\circ}$ on the primary component and generated synthetic profiles for three chosen orbital inclinations: $i=87.2^{\circ}$ (the value obtained from simultaneous LC and RV analysis), $80^{\circ}$, and $70^{\circ}$. Synthetic profiles were generated using the data sampling of La Palma observations to test the effects of phase coverage. The results are shown in Fig. 17. It is clear from the top panel of Fig. 17 that the resultant map shows a high-latitude spot located at latitude of $\sim 60^{\circ}$ together with a symmetric counterpart on the southern hemisphere. The surface map shown in the middle panel of Fig. 17 shows a 'polar like' spot, a feature having a doublepeaked filling factor $\left(f_{\mathrm{s}}\right)$ distribution between $\sim 70^{\circ}$ and $90^{\circ}$, while the lower panel clearly exhibits a polar spot with spot coverage peaking at $90^{\circ}$. Furthermore, the effect of poor phase sampling (between $\sim 210^{\circ}$ and $360^{\circ}$ ) is obviously seen in all surface maps. These results indicate that the existence of polar caps has to be taken into account with some care as one approaches $90^{\circ}$, since this may be due to a maximum entropy effect whereby it is less penalizing to add a high-latitude band rather than a complete polar spot. Therefore, while the mid-low latitude structure is reliable, we cannot rule out a polar spot instead of the high latitude spots seen on our maps of the primary component. It is also a known phenomenon that the spots near the equator have smearing and vertical elongation effects as a consequence of poor latitude discrimination of Doppler imaging around the equator, especially for stars with high inclinations and poor data sampling (Collier-Cameron \& Unruh 1994). Such features are visible in the surface map obtained from Calar Alto data at around longitudes $0^{\circ}-30^{\circ}$ and $300^{\circ}-330^{\circ}$, while similar features are also visible in the surface map obtained using La Palma data between longitudes $210^{\circ}$ and $360^{\circ}$. In the surface map based on the $\mathrm{La}$ Palma data set, where we have good sampling between longitudes $0^{\circ}$ and $200^{\circ}$, low- to mid-latitude spots together with the high latitude (or polar) ones are obvious. The two images separated by about 1 year are qualitatively similar, with a strong high-latitude (or nearpolar) spot complex and scattered low-latitude spots with a smaller filling factor that spread over the entire surface. Polar spots on active cool stars can live very long (Hussain 2002; Strassmeier 2009) possibly owing to emergence of bipolar magnetic regions at very high latitudes and with large tilt angles (Işık, Schüssler \& Solanki 2007), but since the polarity information is not available here, we cannot discuss the details about magnetic structure and evolution of these features. We also performed additional simulations to determine the orbital inclination limit that causes the reconstruction of high-latitude spots rather than polar ones as well as the vertical elongation, for SV Cam configuration. The results showed that the input polar spot becomes a high-latitude band and the vertical elongation begins when the orbital inclination is $i=85^{\circ}$.

\subsubsection{Light-curve inversions and numerical tests on latitudinal dependence}

Fig. 15 shows the surface map obtained using $R$-band light curve of the system with two main spot features centred at around longitudes $\sim 50^{\circ}$ and $300^{\circ}$, which are also in accordance with the location of spots estimated via PHOEBE (Section 5). Numerical tests using the DOTS code with the parameters of RS CVn-type binaries have shown that the longitudinal accuracy of the reconstructions is sensitive to the accuracy of the system parameters such as effective temperatures and radii of components (Jeffers et al. 2005; Özavc1 et al. 2018). We note that in our $R$-band light-curve inversion, the system parameters of SV Cam are preset to values obtained from the simultaneous light and radial velocity solution, so we do not expect in our inversion persistent, spurious spots near quadrature phases related to random background spots (see Özavc1 et al. 2018, section 3.2).

The symmetry of spots with respect to the equator is again a consequence of a high orbital inclination. Normally, it is not possible to obtain latitudinal information from photometry, because a light curve represents one-dimensional time series, hence the resulting map contains only the longitudinal spot distribution information (Berdyugina 2005). However, the resultant map from our light-curve inversion shows latitudinal structuring, especially around latitude $30^{\circ}$.

In order to investigate this pattern, we performed numerical tests. We generated four sets of synthetic spotted light curves using the same system parameters and the data sampling of SV Cam light curves. We put two spots with the same size $\left(r=20^{\circ}\right)$ and contrast. We fixed the longitudes to represent our map of SV Cam, as $\lambda_{S p 1}$ $=50^{\circ}$ and $\lambda_{s p 2}=300^{\circ}$. We considered four cases for the spot 

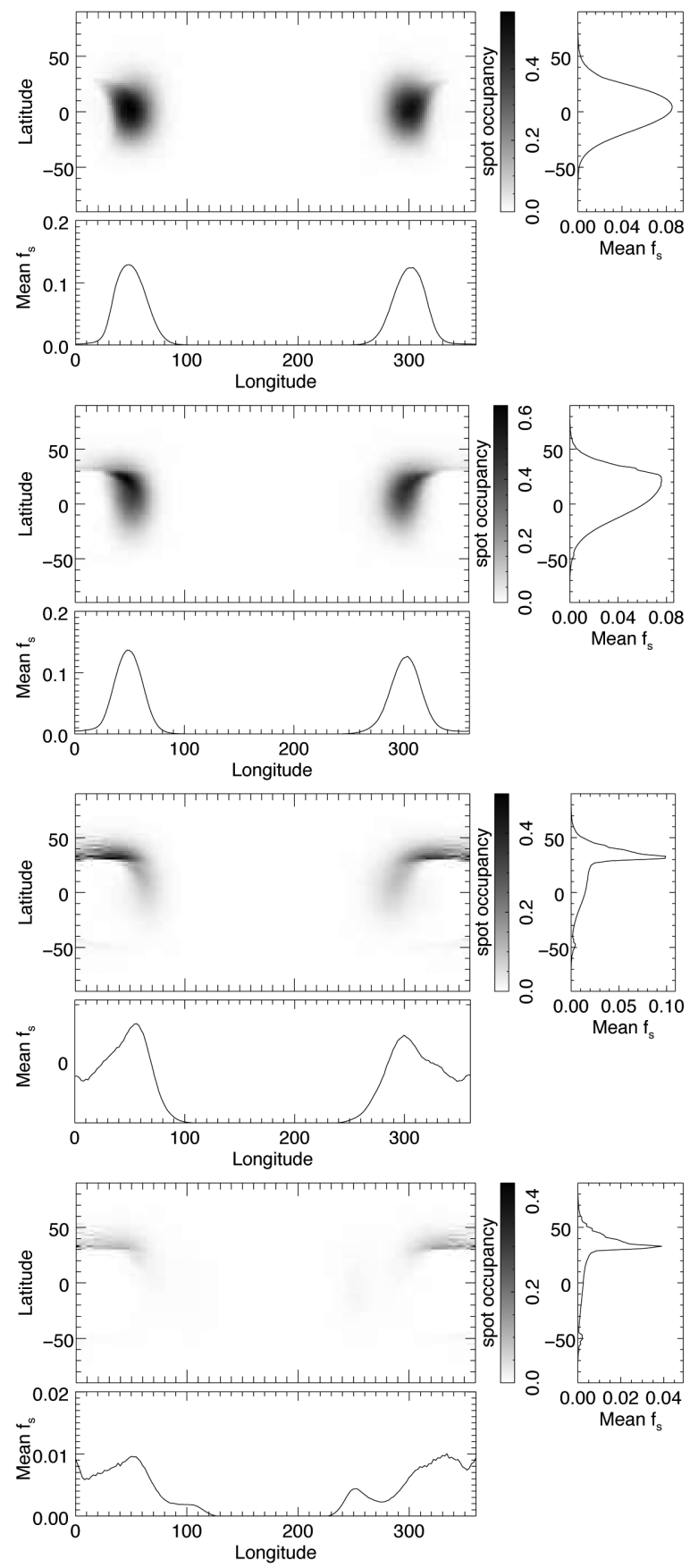

Figure 18. Test results concerning the reconstruction of spots with different latitudes from top to bottom: $0^{\circ}, 20^{\circ}, 60^{\circ}$, and $80^{\circ}$.

latitude: $\varphi_{\text {case } 1}=0^{\circ}, \varphi_{\text {case } 2}=20^{\circ}, \varphi_{\text {case } 3}=60^{\circ}$, and $\varphi_{\text {case } 4}=80^{\circ}$. The reconstructed images are shown in Fig. 18. With increasing input latitude, the reconstructed spots become more asymmetric about the equator, as a consequence of vertical elongation for orbits with high inclination. For $\varphi \geq 20$, a sharp peak starts to appear at around latitude $30^{\circ}$, where the limb of the secondary component traces the boundary with the occulted part of the primary. Our $R$ band reconstruction in Fig. 15 resembles a linear combination of the cases 2 and 3, indicating that both high- and low-latitude spots are present on the primary star. This experiment demonstrates that the structure as well as the latitudinal distribution of reconstructed spots may potentially be used to constrain the latitudes of spots on the eclipsed components of binary systems (Collier Cameron 1997).

\subsection{Chromospheric activity}

We were able to reconstruct the surface map of the primary component only, since the contribution of the secondary component to the total flux is considerably low $(\sim 11$ per cent, depending on the orbital phase and wavelength). However, the high activity at the chromospheres of both components of SV Cam is also known from the literature (e.g. Montes et al. 1995; Pojmanski 1998; Özeren et al. 2001; Kjurkchieva et al. 2002). Therefore, thanks to the wide wavelength coverage and good quality of our spectra, we were also able to investigate the behaviour of the chromospheric activity in SV Cam by means of the spectral subtraction technique. This method, which is simply based on the comparison of active (target) and non-active (standard) star spectra, was first suggested and applied by Barden (1985). In the optical wavelength range, it can be successfully applied to chromospheric activity indicators such as the CaII H\&K, $\mathrm{H} \alpha$, and $\mathrm{H} \beta$ lines, obtaining chromospheric line emission features as residuals.

\subsubsection{Spectral synthesis}

Since SV Cam is an eclipsing binary, the spectra of the system include flux contribution from both components whose intensity and RV depend on the orbital phase. The variations in RV and flux contribution must be taken into account when constructing a non-active template spectrum. To this purpose, we improved a simple light-curve model based on the equations given by Dan Bruton $^{2}$ to calculate the visible area of each component seen by the observer throughout the orbital phase. This simple model assumes spherical components revolving around circular orbits and neglects limb / gravity darkening effects. For the computations, we used the physical parameters of SV Cam $\left(M_{1,2}, R_{1,2}, R_{1,2} a, i\right)$, which we derived from the simultaneous light and RV analysis of the system. The wavelength-dependent contribution of each component to the total continuum is calculated using the parameter:

$\alpha:=\left(\frac{R_{1}}{R_{2}}\right)^{2}\left(\frac{B_{1}(\lambda)}{B_{2}(\lambda)}\right)\left(\frac{A_{1}(\phi)}{A_{2}(\phi)}\right)$,

where $R_{1,2}$ are the radii, $B_{1,2}(\lambda)$ are the Planck functions, and $A_{1,2}(\phi)$ represent the fractional projected area of each component as seen by the observer at phase $\phi$ ( 0 when fully eclipsed, 1 for outside eclipse). Consequently, the weights for the primary and secondary components $\left(W_{1,2}\right)$ are given by

$W_{1}=\frac{\alpha}{1+\alpha} ; \quad W_{2}=\frac{1}{1+\alpha}$,

which are represented in Fig. 19 for the $\mathrm{H} \alpha$ region.

We performed spectral subtraction to La Palma data only, because it has a better phase coverage, allowing a more precise RV solution. To represent the non-active contribution of SV Cam, we used the spectra of $18 \mathrm{Sco}(\mathrm{G} 2 \mathrm{~V})$ and $61 \mathrm{CygA}(\mathrm{K} 5 \mathrm{~V})$ as low-activity templates for the primary and the secondary components, respectively. In fact, $61 \mathrm{Cyg} \mathrm{A}$ is known to be a moderately active star (Duncan et al. 1991). Boro Saikia et al. (2016) reported the presence of a possible magnetic cycle that is twice the length of the activity cycle and also observed polarity reversals. On the other hand, $61 \mathrm{Cyg} \mathrm{A}$

${ }^{2}$ http://www.physics.sfasu.edu/astro/ebstar/ebstar.html 


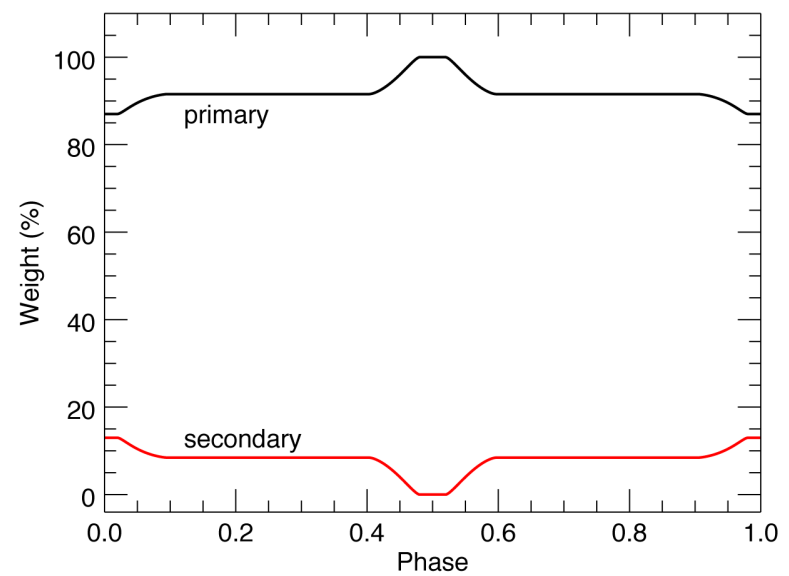

Figure 19. The weights of each component of SV Cam in the $\mathrm{H} \alpha$ region as a function of the orbital phase. The black solid line represents the weight of the primary component, while red solid line shows that of the secondary component.

has been used as a template star several times in the literature for spectral subtraction technique (see, e.g. Martínez-Arnáiz et al. 2010; Alonso-Floriano et al. 2015). It is also considered as a low-activity star (see Table 2 of Martínez-Arnáiz et al. 2010), in comparison with other similar mid-K type dwarf stars. The vsini value of $61 \mathrm{Cyg} \mathrm{A}$ is $\sim 1.1 \mathrm{~km} \mathrm{~s}^{-1}$ (Marsden et al. 2014). During our spectral synthesis of SV Cam, the spectra are broadened at the vsini value of the secondary component of SV Cam, $69 \mathrm{~km} \mathrm{~s}^{-1}$. Therefore, the effect of activity in the core of the $\mathrm{H} \alpha$ and $\mathrm{Ca}$ II IRT lines become much lower and may be considered as negligible. Since we compare the variation of photospheric (DI) and chromospheric activity (spectral synthesis) of SV Cam, the effect of the activity on the $\mathrm{H} \alpha$ and $\mathrm{Ca}$ II IRT lines of $61 \mathrm{Cyg}$ A only leads to an offset on the equivalent width axis. This does not affect the essence of our results, as we do not intend to provide standardized equivalent widths of SV Cam.

The spectra of $18 \mathrm{Sco}$ and $61 \mathrm{CygA}$ were also obtained during La Palma observing run. The weights for the two components at the phases of SV Cam observations were calculated using the relative flux contribution mentioned above (equations 1 and 2). The rotational equatorial velocities were calculated assuming rotation periods equal to the orbital one (synchronous rotation) for both components and the radii listed in Table 2. We obtained $V_{\mathrm{eq}}^{1}$ $=112 \mathrm{~km} \mathrm{~s}^{-1}$ and $V_{\mathrm{eq}}^{2}=69 \mathrm{~km} \mathrm{~s}^{-1}$ for the primary and secondary component, respectively. The spectral subtraction method also requires a careful normalization process. Since the standards used for the spectral subtraction were also obtained with the HERMES spectrograph and the data reduction was performed with the automatic pipeline, the normalization of each selected chunk including the activity indicator lines is easily performed, using second-order polynomial fits. To test the consistency of the spectral synthesis with the measured radial velocities, the calculated relative weights, and vsini values of both components, we first modelled a spectral region which includes mostly photospheric lines (between 5560 and $5650 \AA$ ). To this purpose, we used the spectrum of SV Cam taken at phase 0.749 , in which the separation between the components is maximum. The resultant fit (total flux) as well as the residuals (subtracted spectrum) are shown in Fig. 20. The match between the observed and synthetic spectra is well enough to apply the spectral subtraction technique.

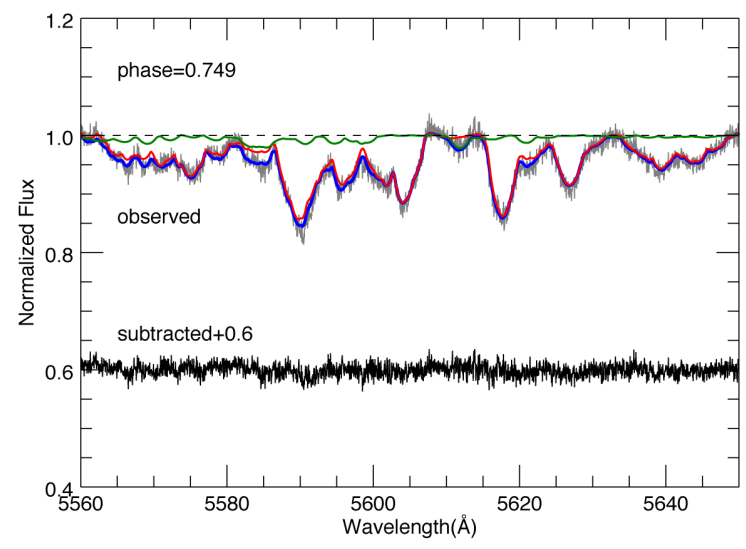

Figure 20. The results of the consistency test for the spectral subtraction method for SV Cam. The grey solid line represents the normalized spectrum of SV Cam corresponding to phase 0.749 . The red and green solid lines show the spectra of the primary and secondary components, respectively, while the blue solid line represents the total flux. The black solid line shows the residuals from the fit.

\subsubsection{Variations in subtracted spectra}

After showing the validity of synthetic spectra, we first investigate the $\mathrm{H} \alpha$ line behaviour of the system with the help of the spectral subtraction technique, using the HERMES data from La Palma. The subtraction process is shown for a sample of phases in Fig. 21. The small amount of contribution of the secondary component to the total flux, along with the rapid rotation of the system prevent us to clearly disentangle the individual contribution of each component. In addition, the maximum wavelength separation between the components is $\sim 6 \AA$, well below the line width of the primary. Therefore, when applying the spectral subtraction technique, we consider the total $\mathrm{H} \alpha$ profile of the system and integrated the area above or below the zero level in the subtracted profile, within \pm 20 $\AA$ about the line centre.

The resulting phase variation of the excess $\mathrm{H} \alpha$ equivalent width is shown in Fig. 22b, where positive and negative values represent excess emission and absorption, respectively.

At the phases of large velocity separation (0.144 and 0.280$)$, an emission feature is clearly visible in the residual spectra (Fig. 21), at the location of the secondary component. This unambiguously demonstrates the high activity level of the K4V-type secondary.

Another interesting feature is the excess absorption seen around phase 0.5 , when the primary star is in front of the secondary, which is clearly displayed in Fig. 22b. Extra absorption in the $\mathrm{H} \alpha$ line has been observed at phases preceding or following the eclipses in other RS CVn stars and has been explained as the effect of prominences projected over the disc of the occulted star, similar to $\mathrm{H} \alpha$ filaments on the Sun (see, e.g. Hall \& Ramsey 1992; Frasca et al. 2000). For SV Cam, a similar excess absorption was reported by Özeren et al. (2001) (see their Fig. 3) between phases 0.619 and 0.744.

Another good indicator of chromospheric activity is the Ca II infrared triplet (Ca II IRT) lines, which have several advantages over the Balmer lines, because Ca II IRT lines are less affected by the telluric absorptions and atmospheric extinction; they also have a well-defined continuum (Frasca et al. 2010). Therefore, we have also investigated the variation of Ca II IRT lines for HERMES data, under the same assumptions considered for $\mathrm{H} \alpha$, but within $\pm 12 \AA$ region. The resulting total variation of Ca II IRT excess emission, i.e. the sum of the excess equivalent widths of the three members of the triplet $(\lambda 8498, \lambda 8542$, and $\lambda 8662)$, is shown in 

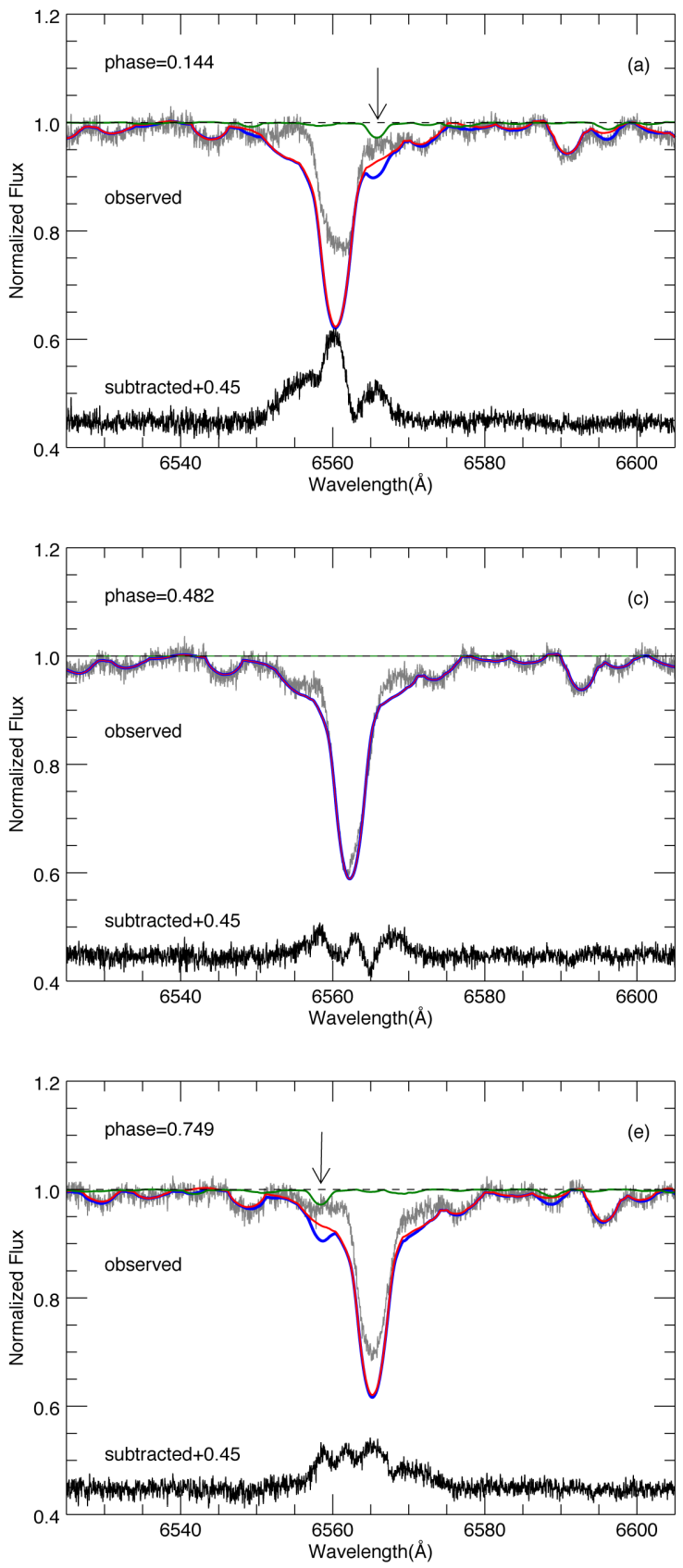
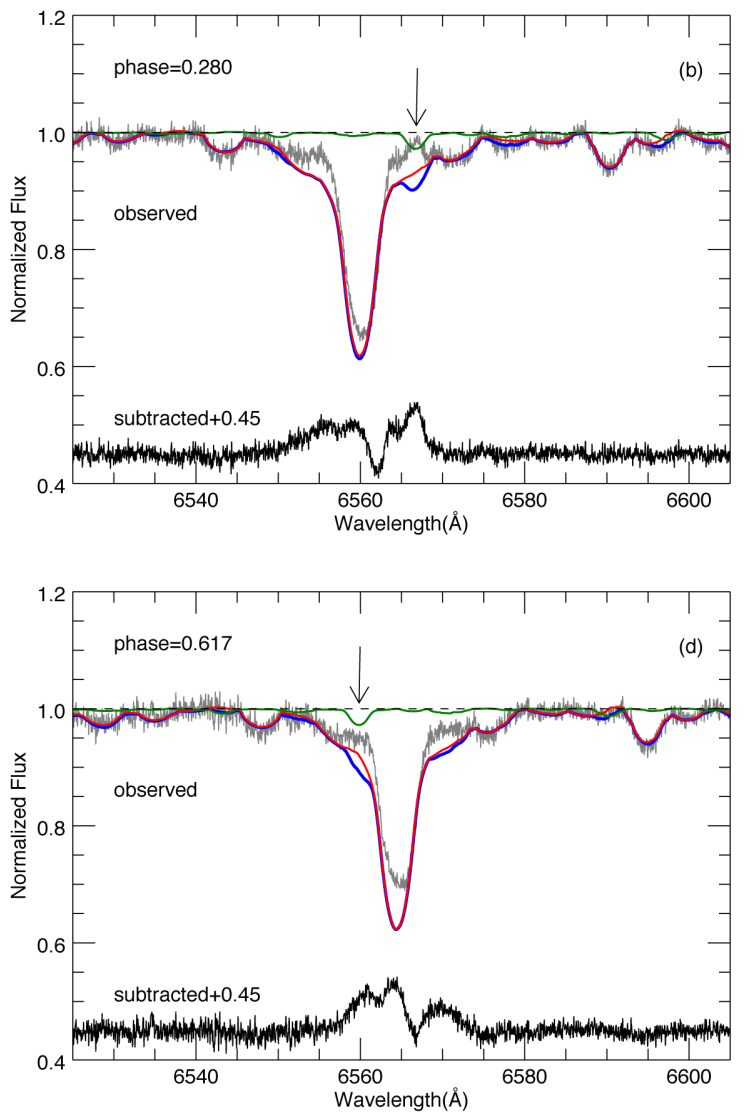

Figure 21. The $\mathrm{H} \alpha$ variation of SV Cam together with the synthetic spectra and the residuals. The arrows show the positions of $\mathrm{H} \alpha$ lines of the secondary component. The colour coding is as same as Fig. 20.

Fig. 22a. The $f_{\mathrm{s}}$ variation along with the orbital phase and the corresponding DI map of the primary component are shown in Figs $22 \mathrm{c}$ and $\mathrm{d}$ to track the spot distribution. Here, the variation of $f_{\mathrm{s}}$ and excess $\mathrm{H} \alpha$ is in accordance with each other especially between phases 0.5 and 1.0, while in the phase range $0.0-0.5$ the $f_{\mathrm{s}}$ values are lower but the excess $\mathrm{H} \alpha$ emission is high. However, we point out that the small number of spectra in this phase range may have caused a poorer DI reconstruction. A comparison of Fig. 22a and Fig. 22b clearly shows that the variation of Ca II IRT lines closely follows the $\mathrm{H} \alpha$ trend, except for two spectra showing excess $\mathrm{H} \alpha$ absorption, obtained during the secondary minimum.

At phases 0.45 and 0.50 , the $\mathrm{H} \alpha$ line shows significant excess absorption, while the IRT maintains its excess emission below the average level. This indicates that the plage contribution from the primary star's chromosphere is more effective in filling up the IRT lines than absorption, whereas the $\mathrm{H} \alpha$ line is very sensitive to relatively cool filaments hanging in the chromosphere. To check whether cool filaments have a measurable effect, we measured the flux ratio of the IRT lines at 8542 to $8498 \AA$ and found that the ratio fluctuates between 1.26 and 1.86 through all orbital phases, with a mean value of 1.46 . These values are indicative of optically thick emission which is likely the result of a dominant contribution of chromospheric plage regions associated with star-spots. This is supported by the spot distribution near the secondary minimum (Fig. 22d), which is not very different than the more densely sampled phases. We conclude that even when there is significant excess 

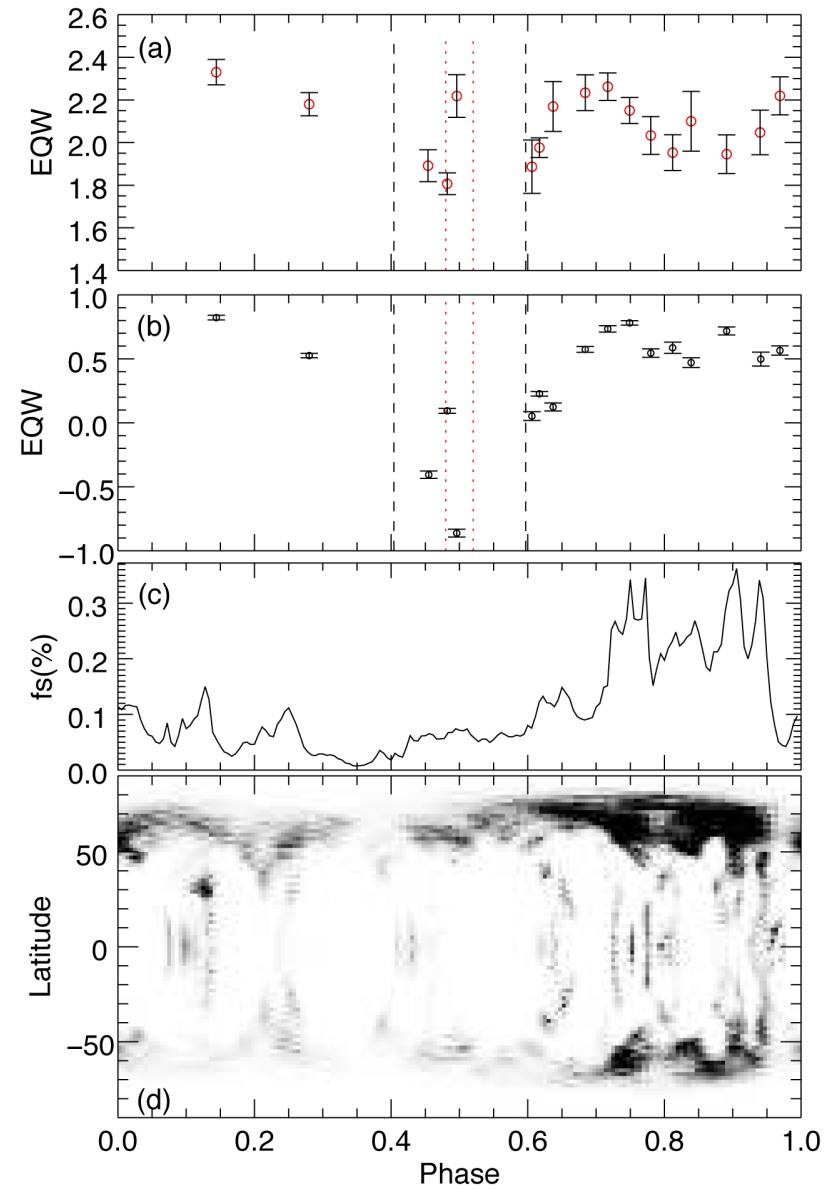

Figure 22. The variation of the equivalent width of Ca II IRT excess emission (a) and $\mathrm{H} \alpha$ excess emission/absorption (b), obtained using the spectral subtraction technique. The black dashed lines show the beginning and the end of the secondary minimum, while the red dotted lines represent the beginning and ending phases of the totality. The spot filling factor $\left[f_{\mathrm{s}}\right](\mathrm{c})$ and the surface map of the primary component of SV Cam (d) obtained from the DI analysis.

absorption in $\mathrm{H} \alpha$, the chromospheric IRT emission integrated over the stellar disc dominates the effect of any optically thin material near the stellar disc.

\section{CONCLUSIONS}

(i) To obtain accurate orbital and physical parameters of the system, we modelled multiband light curves with high precision and short cadence, simultaneously with radial velocity data, which required spot modelling during the analysis. The best fit system parameters are consistent with our photometric mapping and Doppler imaging results using the DOTs code.

(ii) The light-curve inversion performed for the $R$-band light curve resulted in two huge spots centred at longitudes $\sim 50^{\circ}$ and $300^{\circ}$ of the primary (F9V). This is consistent with the simultaneous multiband light curve and RV solution. The inverted map of the primary component indicates that the strongest activity in 2016.00 was concentrated around the phases where the primary is facing the secondary component.

(iii) The primary component of SV Cam shows a high coverage of spots, which can be found at all longitudes. Though the DI maps show that the spots on the primary component are concentrated at high latitudes excluding the poles, the numerical tests concerning the orbital inclination sensitivity of DI hints that they could actually be polar spots. The small amount of flux contribution of the secondary component prevents us from obtaining the DI maps of that companion.

(iv) The spectral synthesis and subtraction applied to this close binary system clearly reveal strong chromospheric activity of the secondary $(\mathrm{K} 4 \mathrm{~V})$ component, though its filling fraction in the systemic radiative flux is very low. The phase variations of $\mathrm{H} \alpha$ and $\mathrm{Ca}$ II IRT lines indicate that the primary (F9V) is covered with strong chromospheric plage regions throughout all the phases, in parallel with spot regions. The excess $\mathrm{H} \alpha$ absorption detected near the secondary eclipse can be led by large cool prominence structures overcoming the background excess emission.

\section{ACKNOWLEDGEMENTS}

The authors are grateful to the anonymous referee for critical comments, which helped to improve the manuscript substantially. HVŞ acknowledges the support by The Scientific And Technological Research Council Of Turkey (TÜBITAK) through the project 1001115F033. DM acknowledges support by the Spanish Ministry of Economy and Competitiveness (MINECO) from project AYA201679425-C3-1-P. AF acknowledges Istituto Nazionale di Astrofisica (INAF) for financial support. EI acknowledges support by the Young Scientist Award Programme BAGEP-2016 of the Science Academy, Turkey.

This paper includes data taken at the Calar Alto, the Roque de los Muchachos observatories and the Astronomical Observatory of the Jagiellonian University.

\section{REFERENCES}

Aceituno J. et al., 2013, A\&A, 552, A31

Albayrak B., Demircan O., Djurašević G., Erkapić S., Ak H., 2001, A\&A, 376,158

Alonso-Floriano F. J. et al., 2015, A\&A, 577, A128

Barden S. C., 1985, ApJ, 295, 162

Barnes J. R., Lister T. A., Hilditch R. W., Collier Cameron A., 2004, MNRAS, 348, 1321

Berdyugina S. V., 2005, Living Rev. Sol. Phys., 2, 8

Boro Saikia S. et al., 2016, A\&A, 594, A29

Collier Cameron A., 1997, MNRAS, 287, 556

Collier-Cameron A., Unruh Y. C., 1994, MNRAS, 269, 814

Djurasevic G., 1998, A\&AS, 127, 233

Donati J.-F., Semel M., Carter B. D., Rees D. E., Collier Cameron A., 1997, MNRAS, 291, 658

Duncan D. K. et al., 1991, ApJS, 76, 383

Frasca A., Marino G., Catalano S., Marilli E., 2000, A\&A, 358, 1007

Frasca A., Biazzo K., Kővári Z., Marilli E., Çakırlı Ö., 2010, A\&A, 518, A48

Gray D. F., 1992, The Observation and Analysis of Stellar Photospheres, Cambridge University Press, Cambridge.

Hall J. C., Ramsey L. W., 1992, AJ, 104, 1942

Hempelmann A., Hatzes A. P., Kuerster M., Patkos L., 1997, A\&A, 317 , 125

Holzwarth V., Schüssler M., 2003, A\&A, 405, 303

Hroch F., 1998, in Dusek J., ed., Proceedings of 20th Stellar Conference of the Czech and Slovak Astronomical Institutes. Nicholas Copernicus Observatory and Planetarium, Brno, Czech Republic, p. 30

Hussain G. A. J., 2002, Astron. Nachr., 323, 349

Işık E., Schüssler M., Solanki S. K., 2007, A\&A, 464, 1049

Işık E., Schmitt D., Schüssler M., 2011, A\&A, 528, A135

Jeffers S. V., Collier Cameron A., Barnes J. R., Aufdenberg J. P., Hussain G. A. J., 2005, ApJ, 621, 425 
Jeffers S. V., Barnes J. R., Collier Cameron A., Donati J.-F., 2006a, MNRAS, 366, 667

Jeffers S. V., Aufdenberg J. P., Hussain G. A. J., Collier Cameron A., Holzwarth V. R., 2006b, MNRAS, 367, 1308

Kjurkchieva D. P., Marchev D. V., Zola S., 2002, A\&A, 386, 548

Kupka F., Piskunov N., Ryabchikova T. A., Stempels H. C., Weiss W. W., 1999, A\&AS, 138, 119

Lucy L. B., 1967, ZAp, 65, 89

Manzoori D., 2016, Astron. Nachr., 337, 652

Marsden S. C. et al., 2014, MNRAS, 444, 3517

Martínez-Arnáiz R., Maldonado J., Montes D., Eiroa C., Montesinos B., 2010, A\&A, 520, A79

Montes D., Fernandez-Figueroa M. J., de Castro E., Cornide M., 1995, A\&A, 294, 165

Özavcı I., Şenavcı H. V., Işık E., Hussain G. A. J., O’Neal D., Yılmaz M., Selam S. O., 2018, MNRAS, 474, 5534

Özeren F. F., Gunn A. G., Doyle J. G., Jevremović D., 2001, A\&A, 366, 202

Patkos L., 1982, Commun. Konkoly Obs. Hungary, 80, 1

Patkos L., Hempelmann A., 1994, A\&A, 292, 119

Pojmanski G., 1998, Acta Astron., 48, 711

Prša A., Zwitter T., 2005, ApJ, 628, 426

Raskin G. et al., 2011, A\&A, 526, A69

Roettenbacher R. M. et al., 2016, Nature, 533, 217

Ruciński S. M., 1969, Acta Astron., 19, 245
Rucinski S., 1999, Turk. J. Phys., 23, 271

Rucinski S. M., Lu W., Capobianco C. C., Mochnacki S. W., Blake R. M., Thomson J. R., Ogłoza W., Stachowski G., 2002, AJ, 124, 1738

Schuessler M., Solanki S. K., 1992, A\&A, 264, L13

Şenavcı H. V., Hussain G. A. J., O’Neal D., Barnes J. R., 2011, A\&A, 529, A11

Strassmeier K. G., 2009, A\&AR, 17, 251

Strassmeier K., Washuettl A., Granzer T., Scheck M., Weber M., 2000, A\&AS, 142, 275

Unruh Y. C., 1996, in Strassmeier K. G., Linsky J. L., eds, IAU Symposium Vol. 176, Stellar Surface Structure, Dordrecht, p. 35

van Hamme W., 1993, AJ, 106, 2096

Xiang Y., Gu S., Cameron A. C., Barnes J. R., 2015, MNRAS, 447, 567

Zboril M., Djurašević G., 2003, A\&A, 406, 193

Zboril M., Djuraševič G., 2004, Contrib. Astron. Obs. Skalnate Pleso, 34, 128

Zboril M., Djuraševič G., 2006, Contrib. Astron. Obs. Skalnate Pleso, 36, 77

Zeilik M., de Blasi C., Rhodes M., Budding E., 1988, ApJ, 332, 293

This paper has been typeset from a $\mathrm{T}_{\mathrm{E}} \mathrm{X} / \mathrm{L} \mathrm{T} \mathrm{E} \mathrm{X}$ file prepared by the author. 